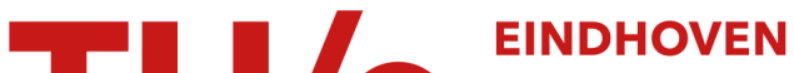 UNIVERSITY OF TECHNOLOGY
}

\section{Temperature-dependent sintering of two viscous particles}

Citation for published version (APA):

Balemans, C., Jaensson, N. O., Hulsen, M. A., \& Anderson, P. D. (2018). Temperature-dependent sintering of two viscous particles. Additive Manufacturing, 24, 528-542. https://doi.org/10.1016/j.addma.2018.09.005

DOI:

10.1016/j.addma.2018.09.005

Document status and date:

Published: 01/12/2018

\section{Document Version:}

Accepted manuscript including changes made at the peer-review stage

\section{Please check the document version of this publication:}

-A submitted manuscript is the version of the article upon submission and before peer-review. There can be important differences between the submitted version and the official published version of record. People interested in the research are advised to contact the author for the final version of the publication, or visit the $\mathrm{DOI}$ to the publisher's website.

- The final author version and the galley proof are versions of the publication after peer review.

- The final published version features the final layout of the paper including the volume, issue and page numbers.

Link to publication

\section{General rights}

Copyright and moral rights for the publications made accessible in the public portal are retained by the authors and/or other copyright owners and it is a condition of accessing publications that users recognise and abide by the legal requirements associated with these rights.

- Users may download and print one copy of any publication from the public portal for the purpose of private study or research.

- You may not further distribute the material or use it for any profit-making activity or commercial gain

- You may freely distribute the URL identifying the publication in the public portal.

If the publication is distributed under the terms of Article $25 \mathrm{fa}$ of the Dutch Copyright Act, indicated by the "Taverne" license above, please follow below link for the End User Agreement:

www.tue.nl/taverne

Take down policy

If you believe that this document breaches copyright please contact us at:

openaccess@tue.nl

providing details and we will investigate your claim. 


\title{
Temperature-dependent sintering of two viscous particles
}

\author{
Caroline Balemans ${ }^{\mathrm{a}, \mathrm{b}}$, Nick O. Jaensson ${ }^{\mathrm{a}}$, Martien A. Hulsen ${ }^{\mathrm{a}}$, and Patrick D. \\ Anderson $^{\mathrm{a}, *}$

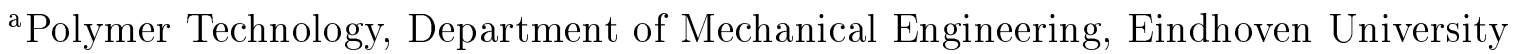 \\ of Technology, 5600 MB Eindhoven, The Netherlands \\ ${ }^{\mathrm{b}}$ Brightlands Materials Center, 6167 RD Geleen, The Netherlands \\ ${ }^{*}$ Corresponding author. E-mail address: P.D.Anderson@tue.nl
}

\begin{abstract}
Selective laser sintering (SLS) is a promising additive manufacturing technique, where powder particles are fused together under the influence of a laser beam. To obtain good material properties in the final product, the powder particles need to form a homogeneous melt during the fabrication process. On the one hand, you want to give the particles enough time to fuse, such that the original shape is no longer visible, and interdiffusion of polymers can take place. On the other hand, you want the process to be as fast as possible. This is contradictory, thus choosing the right conditions is not trivial. We developed a computational model based on the finite element method to study the material and process parameters concerning the melt flow of the powder particles. In this work, we restrict ourselves to varying the temperature-dependent viscosity, the process parameters, and the convective heat transfer coefficient of the sintering of two polymer (polyamide 12) particles. The simulations allow for a quantitative analysis of the influence of the different material and processing parameters. From the simulations follows that an optimal sintering process has a low ambient temperature, a narrow beam width with enough power to heat the particles only a few degrees above the melting temperature, and a polymer of which the viscosity decreases significantly within these few degrees.
\end{abstract}

Keywords: finite element method; temperature-dependent viscous flow; coalescence; 3D printing; SLS

\section{Introduction}

Selective laser sintering (SLS) is an additive manufacturing method which uses a laser to selectively sinter powder, layer-by-layer, to produce three dimensional objects. The materials used as powder in SLS are metals, ceramics, glasses, and polymers. Advantages of the SLS fabrication technique are geometrical freedom and cheap prototyping. However, product quality cannot be guaranteed yet. One of the mechanisms improving the final product quality is the formation of a homogeneous melt during the fabrication process, giving the particles enough time to fuse. We design a computational model to describe the sintering process in detail and vary the material and process parameters that play an important role in the melt flow of the powder.

Many articles report on the mechanisms of sintering and heat transfer of metal powder. Kou and Wang [1] simulated the convection in laser melted pools of an aluminum alloy, and considered the buoyancy force and surface tension gradient at the weld pool 
surface as driving forces. Tolochko et al. [2] studied the sintering of titanium powder, and used Kingery's [3] solid state sintering mechanisms to describe the kinetics. Patil and Yadava [4] presented a thermal model to predict the temperature distribution in a titanium layer, including the effect of the laser beam's Gaussian heat flux, the effective thermal conductivity, the powder bed density, and the temperature-dependent thermal properties of the material. Chen and Zhang $[5,6]$ described the sintering process of a two-component metal powder layer on top of multiple existing sintered layers. In their work, the velocities of the liquid phase were obtained by Darcy's law. Roberts et al. [7] predicted the transient temperature field for multiple layers using the finite element method, where the laser energy absorption including the non-linearities produced by both the material properties and phase changes are taken into account. Furthermore, Kholpanov et al. [8] and Zakiev et al. [9] made models to describe simultaneous self-propagating high-temperature synthesis and selective laser sintering of powder compositions. Kolossov et al. [10] used the finite element method to describe the temperature evolution and formation process of sintered parts, where Frenkel's [11] expression of the sintering rate is used to define the sintering potential.

However, the sintering of polymer particles is expected to be significantly different, e.g., due to different thermal properties of the polymeric material. Frenkel's [11] approach to determine the sintering rate of the model structure is used by Scherer [12] as well. He found a model to describe the evolution of density in the sintering process of low-density glasses. Kandis and Bergman [13] used Scherer's definition to model the manufacturing of multiple layer samples of amorphous polymers. Sun [14] extended Scherer's model and developed a method together with Beamann [15] to calculate the temperature and density distribution of a powder bed where the material properties and the laser control parameters are given as input. Subsequently, Papadatos et al. [16] used this work to characterize and model the phenomenon where material properties in $z$-direction differ from those in the rest of the part due to a non-homogeneous sintering. Furthermore, Scherer's model is used in combination with the model proposed by Mackenzie and Shuttleworth [17] to describe the evolution of density of amorphous polymers: Nelson et al. [18] used the definition for the local densification rates to model the behavior of polycarbonate powder beds, Bugeda et al. [19] found a new strategy to rewrite the thermal and sintering equations in order to get a steady state problem, and Singh and Srinivasa Prakash [20] predicted density variations in the sintered parts in terms of process variables such as laser power, scan spacing, and scan velocity. Childs et al. [21] simulated the sintering of polycarbonate using adaptive mesh finite difference and fixed mesh finite element methods, and followed the viscous sintering law for variation in density of Nelson, whereupon Dong et al. [22] used Childs' rate of evolution of the density of the powder bed. In later work, Tontowi and Childs [23] expanded Childs' model to predict the density of crystalline polymer sintered parts. In addition to this list of studies, we present in this paper the first detailed analysis of the thermal and flow behavior of two fusing particles.

Generally, two different methods are used to add the laser contribution to the system of equations. In the first method, a surface flux is prescribed on the top surface of the layer as a boundary condition. The second procedure introduces a volumetric source term to the energy balance. The review article of Zeng et al. [24] noted that in most articles the first method is used, since there is limited research done on laser beam penetration. According to Kolossov et al. [10], the heat source can be considered as a surface heat source if the element size of the finite element mesh is larger than five particle diameters. For the various 1D, 2D, and 3D problems in literature, different definitions of the laser contribution are used. One of the most elaborate ones is used by Sun and Beaman [15] and takes the effective absorptivity, the extinction coefficient and the movement of the Gaussian beam into account. Additional boundary conditions at the top surface of the layer usually describe either convection, radiation, or a combination of these two processes. At the bottom of the powder bed, assumed to be at infinite depth, the heat flux is often 
taken zero.

In this work, we study the temperature-dependent SLS process on a smaller scale than the usual powder beds with melt pools. We use a 2D geometry of two circular particles, that are heated up by a volumetric laser source term. The viscous flow and temperature problem are solved using the finite element method. We assess the effect of the temperature-dependent viscosity, the tunable process parameters, and the convective heat transfer coefficient on the fusion of the particles.

\section{Problem description and governing equations}

We study the two-dimensional geometry of two circular liquid polymer particles. These particles are of equal size and are initially connected to each other by a contact line $L(t=0)$. The geometry is shown in Figure 1. The initial domain $\Omega(t=0)$ is defined by the radii of the particles $R$, and the initial contact line $L(t=0)=L_{\mathrm{p}}+L_{\epsilon}$, where $L_{\mathrm{p}}$ is chosen to be $0.4 R$, and $L_{\epsilon}$ appears due to the round off of the contact region using parameter $R_{\mathrm{L}}=R\left(L_{\mathrm{p}} / R\right)^{3}$ [25] to avoid discontinuities in the slope of the interface. Curve $\Gamma$ is the outer boundary of the domain, with $\boldsymbol{n}$ the outwardly directed unit normal vector. The particles and surroundings are preheated to $T(t=0)=T_{\infty}$, with $T_{\infty}$ the ambient temperature, just below the melting temperature. A 2D Gaussian laser beam heats up the particles above the melting point, lowering the viscosity. It is assumed that the viscosity follows an Arrhenius relation and is only a function of temperature $\mu(T)$. Due to the decrease in viscosity, the particles start to flow. Under the influence of the surface tension $\gamma$, which is prescribed on boundary $\Gamma$, the two liquid particles will merge into one larger circular object. Heat losses at the boundary $\Gamma$ reduce the temperature in the system. If the maximum temperature reaches the melting temperature $T_{\mathrm{m}}$, the particles solidify instantaneously.

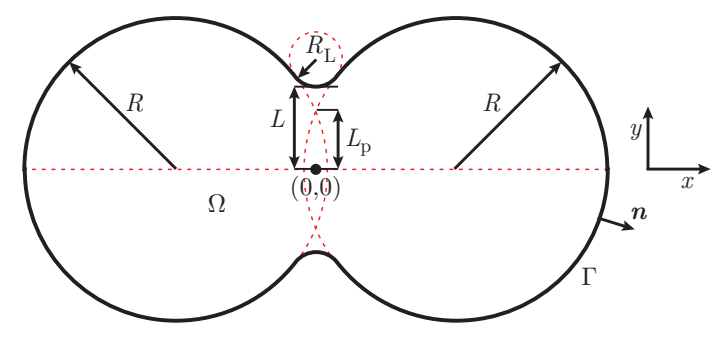

Figure 1: Domain $\Omega(t=0)$ of the two liquid polymer particles.

\subsection{Balance equations and constitutive model}

The sintering process of viscous particles can be described using the momentum balance, mass balance, and energy balance. We assume that the fluid is incompressible, and that inertia and body forces can be neglected [26]. This leads to the following set of equations

$$
\begin{aligned}
-\nabla \cdot(-p \boldsymbol{I}+2 \mu(T) \boldsymbol{D}) & =0 & & \text { in } \Omega \\
\nabla \cdot \boldsymbol{u} & =0 & & \text { in } \Omega \\
\rho c_{\mathrm{p}} \frac{\mathrm{D} T}{\mathrm{D} t} & =k \nabla^{2} T+2 \mu(T) \boldsymbol{D}: \boldsymbol{D}+Q(x, y, t) & & \text { in } \Omega
\end{aligned}
$$

where $\mathrm{D}() / \mathrm{D} t$ denotes the material derivative, $p$ is the pressure, $\mu(T)$ the temperaturedependent viscosity, $\boldsymbol{u}$ the velocity, $\rho$ the density, $c_{\mathrm{p}}$ the specific heat, $T$ the temperature, 
Table 1: Viscosity parameters for PA2200.

\begin{tabular}{llll}
\hline Parameter & Symbol & Value & Reference \\
\hline Activation energy & $E_{\mathrm{a}}$ & $50 \cdot 10^{3} \mathrm{~J} / \mathrm{mol}$ & {$[27]$} \\
Arrhenius coefficient & $\mu_{0}$ & $2 \cdot 10^{-3} \mathrm{~Pa} \cdot \mathrm{s}$ & {$[27]$} \\
Melt temperature & $T_{\mathrm{m}}$ & $187^{\circ} \mathrm{C}$ & {$[28]$} \\
\hline
\end{tabular}

$t$ the time, $k$ the thermal conductivity, $Q$ a source term which will be defined later, and $\boldsymbol{D}=\left(\nabla \boldsymbol{u}+(\nabla \boldsymbol{u})^{\mathrm{T}}\right) / 2$.

The viscosity depends on the temperature according to an Arrhenius relation [27]

$$
\mu(T)=\mu_{0} \exp \left(\frac{E_{\mathrm{a}}}{G T}\right),
$$

where $\mu_{0}$ is a constant coefficient, $E_{\mathrm{a}}$ the activation energy, $G$ the universal gas constant, and $T$ is given in Kelvin. For temperatures below the melting temperature $T_{\mathrm{m}}$, the viscosity goes to infinity

$$
\mu\left(T<T_{\mathrm{m}}\right) \rightarrow \infty
$$

We assume that all other material properties are independent of temperature.

Polyamide 12 (PA12) is used most often in SLS. A widely used sinter powder has the trade name PA2200 and is manufactured by EOS GmbH [27]. In this work, we use the parameters of the Arrhenius relation of PA2200 as given in Table 1 and Figure 2.

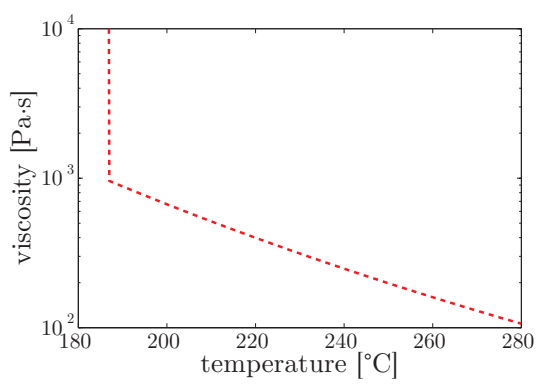

Figure 2: Temperature-dependent viscosity of PA2200.

\subsection{Interface tracking}

We track the motion of the boundary $\Gamma$ in a Lagrangian based way. The velocity of the boundary is defined as

$$
\frac{d \boldsymbol{x}_{\Gamma}}{d t}=\boldsymbol{u},
$$

where $\boldsymbol{x}_{\Gamma}$ are the coordinates of the boundary, and $\boldsymbol{u}$ is the velocity at the boundary $\Gamma$.

\subsection{Boundary and initial conditions}

A constant surface tension $\gamma$ is prescribed on boundary $\Gamma$ using a Neumann boundary condition

$$
(-p \boldsymbol{I}+2 \mu(T) \boldsymbol{D}) \cdot \boldsymbol{n}=\nabla_{\mathrm{s}} \cdot\left(\gamma \boldsymbol{I}_{\mathrm{s}}\right)-p_{\text {out }} \boldsymbol{n} \quad \text { on } \Gamma,
$$


where $\nabla_{\mathrm{s}}$ is the surface gradient operator, $\boldsymbol{I}_{\mathrm{s}}=\boldsymbol{I}-\boldsymbol{n} \boldsymbol{n}$ the second-order unit surface dyadic tensor, and $p_{\text {out }}$ the outside pressure. Since we assume that the fluid is incompressible and the material properties are independent of pressure, the outside pressure does not play a role. Therefore, we assume $p_{\text {out }}=0$ in the rest of the manuscript. To prevent rigid body motion, we fix the origin $(0,0)$ in both $x$ - and $y$-direction and point $(0, L)$ in $x$-direction

$$
\begin{aligned}
\boldsymbol{u}=0 & \text { at }(0,0), \\
u_{x}=0 & \text { at }(0, L) .
\end{aligned}
$$

Furthermore, we assume that the particles are subject to heat loss due to convection and radiation from the entire boundary $\Gamma$, according to

$$
-k \nabla T \cdot \boldsymbol{n}=h\left(T-T_{\infty}\right)+\sigma_{\mathrm{B}} \varepsilon\left(T^{4}-T_{\infty}^{4}\right) \quad \text { on } \Gamma,
$$

with $h$ the convective heat transfer coefficient, or short 'convection coefficient', $T_{\infty}$ the ambient temperature, $\sigma_{\mathrm{B}}$ the Stefan-Boltzmann constant, and $\varepsilon$ the surface emissivity.

The temperature of the domain $\Omega$ is initially assumed to be equal to the ambient temperature $T(t=0)=T_{\infty}$.

\subsection{Source term}

We assume that the intensity $I(x)$ of the 2D laser beam, see Figure 3, is described using a Gaussian distribution in $x$-direction

$$
I(x)=I_{0} \exp \left(-2 \frac{\left(x-x_{\mathrm{b}}\right)^{2}}{W^{2}}\right)
$$

where $I_{0}$ is the peak intensity, $x_{\mathrm{b}}$ the $x$-coordinate of the center of the incident beam, and $W$ is the distance from the beam axis where $I(W)=I_{0} \exp (-2)$. In this work, we call $W$ the 'beam width' and assume that it is constant. The peak intensity $I_{0}$ of the $2 \mathrm{D}$ laser depends on the laser power $P$, defined per unit length, according to

$$
I_{0}=\frac{P}{\sqrt{\pi W^{2} / 2}} .
$$

The intensity decay in $y$-direction inside the material behaves according to the BeerLambert law

$$
\hat{I}(x, y)=I(x) \exp \left(-\alpha\left(y_{\Gamma}-y\right)\right)
$$

where $\alpha$ is the absorption coefficient, $\left(y_{\Gamma}-y\right)$ is the distance in $y$-direction to the boundary $\Gamma$, with $y_{\Gamma} \geq 0$, and refraction is neglected. The source term $\hat{Q}(x, y)$ is calculated using

$$
\hat{Q}(x, y)=\alpha \hat{I}(x, y)
$$

The complete source term $Q(x, y)$ is

$$
Q(x, y)=\alpha\left(1-\zeta_{\mathrm{R}}\right) \frac{P}{\sqrt{\pi W^{2} / 2}} \exp \left(-2 \frac{\left(x-x_{\mathrm{b}}\right)^{2}}{W^{2}}\right) \exp \left(-\alpha\left(y_{\Gamma}-y\right)\right)
$$

where $\zeta_{\mathrm{R}}$ is the surface reflectivity. We assume that no multiple reflections occur from the curved boundary. 


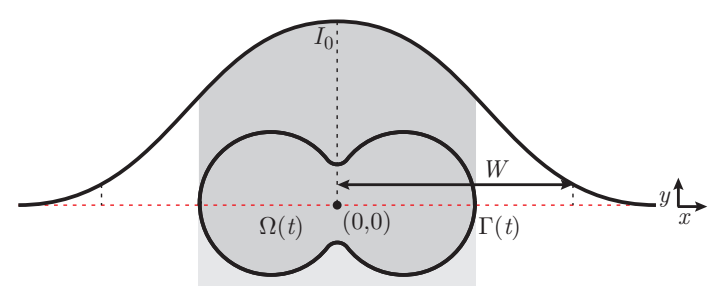

Figure 3: Schematic of the 2D Gaussian laser beam.

\subsection{Dimensionless equations}

The governing equations can be scaled using characteristic values from the problem parameters. We introduce a characteristic length $x_{\mathrm{c}}=R$, a characteristic temperature difference $\Delta T_{\mathrm{c}}=P f_{\mathrm{e}} t_{\mathrm{b}} /\left(2 A \rho c_{\mathrm{p}}\right)$, with $f_{\mathrm{e}}$ the fraction of the laser power $P$ that is effectively used inside the domain, $t_{\mathrm{b}}$ the heating time, and $A$ the area of the domain, a characteristic velocity $u_{\mathrm{c}}=\gamma / \mu_{\mathrm{c}}$, with $\mu_{\mathrm{c}}$ the viscosity at characteristic temperature $T_{\mathrm{c}}=T_{\infty}+\Delta T_{\mathrm{c}}$, a characteristic pressure $p_{\mathrm{c}}=\gamma / R$, and a characteristic time $t_{\mathrm{c}}=R \mu_{\mathrm{c}} / \gamma[26]$. By dividing the original variable by the characteristic value, the dimensionless variable is obtained, e.g.,

$$
x^{*}=\frac{x}{x_{\mathrm{c}}}=\frac{x}{R} .
$$

Herein, the dimensionless variable is represented by an asterisk superscript.

Scaling Eqs. (1) to (3) leads to

$$
\begin{aligned}
\nabla^{*} p^{*}-\left(\nabla^{* 2} \boldsymbol{u}^{*}+\left(\nabla^{* 2} \boldsymbol{u}^{*}\right)^{\mathrm{T}}\right) & =0 & & \text { in } \Omega, \\
\nabla^{*} \cdot \boldsymbol{u}^{*} & =0 & & \text { in } \Omega, \\
\operatorname{Pe} \frac{\mathrm{D} T^{*}}{\mathrm{D} t^{*}} & =\nabla^{* 2} T^{*}+2 \operatorname{Br}\left(\boldsymbol{D}^{*}: \boldsymbol{D}^{*}\right)+\mathrm{N}_{1} Q^{*} & & \text { in } \Omega,
\end{aligned}
$$

where $\mathrm{Pe}=\rho c_{\mathrm{p}} \gamma R /\left(\mu_{\mathrm{c}} k\right)$ is the Péclet number, which is the ratio between the rate of heat advection and the rate of heat conduction, $\mathrm{Br}=\gamma^{2} /\left(\mu_{\mathrm{c}} k \Delta T_{\mathrm{c}}\right)$ is the Brinkman number, which is the ratio of heat produced by viscous dissipation to heat transported by conduction, and $\mathrm{N}_{1}=R^{2}\left(1-\zeta_{\mathrm{R}}\right) \alpha P /\left(k \Delta T_{\mathrm{c}} \sqrt{\pi W^{2} / 2}\right)$ is the ratio between the heat source and the heat conduction. Furthermore

$$
Q^{*}=\exp \left(-2 \mathrm{~N}_{2}\left(x^{*}-x_{\mathrm{b}}^{*}\right)^{2}\right) \exp \left(-\mathrm{N}_{3}\left(y_{\Gamma}^{*}-y^{*}\right)\right),
$$

where $\mathrm{N}_{2}=R^{2} / W^{2}$ is the ratio between the length scale of the system and the length scale of the laser sheet squared, and $\mathrm{N}_{3}=R \alpha$ is the ratio between the length scale of the system and the penetration depth of the laser.

The scaled version of Eq. (4) becomes

$$
\frac{\mu}{\mu_{\mathrm{c}}}=\exp \left(\frac{E_{\mathrm{a}}}{G T}-\frac{E_{\mathrm{a}}}{G T_{\mathrm{c}}}\right) .
$$

Scaling Eq. (10) results in the following equation

$$
-\nabla^{*} T^{*} \cdot \boldsymbol{n}=\mathrm{Nu}^{*}+\mathrm{N}_{4} T^{*},
$$

where $\mathrm{Nu}=h R / k$ is the Nusselt number, which is the ratio of convective heat transport to conductive heat transport, and $\mathrm{N}_{4}=R \sigma_{\mathrm{B}} \varepsilon 4 T_{\infty}^{3} / k$ is the ratio between radiative heat transport and conductive heat transport. Note that for this expression, the linearization $\left(T^{4}-T_{\infty}^{4}\right) \approx 4 T_{\infty}^{3}\left(T-T_{\infty}\right)$ is used. 


\section{$3 \quad$ Numerical method}

We solve Eqs. (1) to (3) with the appropriate boundary conditions using the finite element method. A prediction of the velocity and viscosity fields are used in both the convective term and viscous dissipation term of the energy balance, while the solution of the temperature field is used to compute the viscosity for the momentum and mass balance. The problem has a higher degree of complexity, since we are solving the governing equations on a moving domain. We use isoparametric, triangular P2/P1 (Taylor-Hood) elements for the velocity/pressure, and isoparametric, triangular P2 elements for the temperature.

\subsection{Time integration}

Following the procedure as described in [26], we solve the system of equations on the moving domain $\Omega$. First, the position of boundary $\Gamma$ is predicted from previous time steps

$$
\hat{\boldsymbol{x}}_{\Gamma}^{n+1}=\boldsymbol{x}_{\Gamma}^{n},
$$

for the first time step, and

$$
\hat{\boldsymbol{x}}_{\Gamma}^{n+1}=2 \boldsymbol{x}_{\Gamma}^{n}-\boldsymbol{x}_{\Gamma}^{n-1},
$$

for all subsequent time steps, with $\hat{\boldsymbol{x}}_{\Gamma}^{n+1}$ the prediction of the boundary position for time $t^{n+1}$, and $\boldsymbol{x}_{\Gamma}^{n}$ and $\boldsymbol{x}_{\Gamma}^{n-1}$ are the boundary positions at time $t^{n}$ and $t^{n-1}$. This is followed by the calculation of the displacement of the fluid mesh from the boundary displacement using a Laplace equation [29]. The mesh velocity is calculated using a first-order backward differencing scheme

$$
\boldsymbol{u}_{\mathrm{m}}^{n+1}=\frac{\boldsymbol{x}_{\mathrm{m}}^{n+1}-\boldsymbol{x}_{\mathrm{m}}^{n}}{\Delta t},
$$

for the first time step, and a second-order backward differencing scheme

$$
\boldsymbol{u}_{\mathrm{m}}^{n+1}=\frac{\frac{3}{2} \boldsymbol{x}_{\mathrm{m}}^{n+1}-2 \boldsymbol{x}_{\mathrm{m}}^{n}+\frac{1}{2} \boldsymbol{x}_{\mathrm{m}}^{n-1}}{\Delta t}
$$

for all subsequent time steps, where $\boldsymbol{u}_{\mathrm{m}}^{n+1}$ is the mesh velocity at time $t^{n+1}$, and $\boldsymbol{x}_{\mathrm{m}}^{n+1}$, $\boldsymbol{x}_{\mathrm{m}}^{n}$, and $\boldsymbol{x}_{\mathrm{m}}^{n-1}$ are the mesh coordinates at time $t^{n}, t^{n+1}$, and $t^{n-1}$, respectively.

Subsequently, the velocity and temperature are predicted from previous time steps using

$$
\begin{aligned}
& \hat{\boldsymbol{u}}^{n+1}=\boldsymbol{u}^{n}, \\
& \hat{T}^{n+1}=T^{n},
\end{aligned}
$$

for the first time step, and

$$
\begin{aligned}
& \hat{\boldsymbol{u}}^{n+1}=2 \boldsymbol{u}^{n}-\boldsymbol{u}^{n-1}, \\
& \hat{T}^{n+1}=2 T^{n}-T^{n-1},
\end{aligned}
$$

for all subsequent time steps. Herein $\hat{\boldsymbol{u}}^{n+1}$ is the prediction of the velocity and $\hat{T}^{n+1}$ the prediction of the temperature for time $t^{n+1}$, and $\boldsymbol{u}^{n}, \boldsymbol{u}^{n-1}, T^{n}$, and $T^{n-1}$ are the velocities and temperatures at time $t^{n}$ and $t^{n-1}$, respectively. The prediction of the temperature $\hat{T}^{n+1}$ is used to determine the viscosity, which is used together with the prediction of the velocity $\hat{\boldsymbol{u}}^{n+1}$ as input for the energy equation. The mixed boundary condition is linearized according to

$$
-k \nabla T \cdot \boldsymbol{n}=\left(h+h_{\mathrm{rad}}\right)\left(T-T_{\infty}\right),
$$


with $h_{\mathrm{rad}}=\sigma_{\mathrm{B}} \varepsilon\left(\hat{T}^{2}+T_{\infty}^{2}\right)\left(\hat{T}+T_{\infty}\right)$. The energy equation and the corresponding boundary conditions are written in a weak form by multiplying with the test function $w$, and are rewritten using partial integration and Gauss' theorem. Discretization is done according to the Galerkin approach on the newly defined domain $\Omega$. This leads to the following weak form: Find $T$ such that

$$
\begin{aligned}
\rho c_{\mathrm{p}}\left(w, \frac{\partial T}{\partial t}+\right. & \left.\left(\boldsymbol{u}-\boldsymbol{u}_{\mathrm{m}}\right) \cdot \nabla T\right)+k(\nabla w, \nabla T)+\left(w,\left(h+h_{\mathrm{rad}}\right) T\right)_{\Gamma} \\
& =(w, \mu(T) \boldsymbol{D}: \boldsymbol{D})+(w, Q)+\left(w,\left(h+h_{\mathrm{rad}}\right) T_{\infty}\right)_{\Gamma}, \quad \text { for all } w
\end{aligned}
$$

using appropriate spaces for $T$ and $w$. Note that $(\cdot, \cdot)$ defines the inner product on $\Omega$ and $(\cdot, \cdot)_{\Gamma}$ defines the inner product on $\Gamma$. To integrate in time, we use a first-order backward Euler scheme for the first time step and a second-order backward differencing scheme for all subsequent time steps.

Next, the momentum and mass balance including the boundary conditions are written in a weak form by multiplying with the test functions $\boldsymbol{v}$ and $q$, and are rewritten using partial integration and Gauss' theorem, and Weatherburn's surface divergence theorem [30]. Again, discretization is done according to the Galerkin approach on domain $\Omega$. The viscosity is calculated in each integration point using the new temperature $T^{n+1}$. This leads to the following weak form: Find $\boldsymbol{u}$ and $p$ such that

$$
\begin{aligned}
\left((\nabla \boldsymbol{v})^{\mathrm{T}}, 2 \mu(T) \boldsymbol{D}\right)-(\nabla \cdot \boldsymbol{v}, p) & =-\left(\left(\nabla_{\mathrm{s}} \boldsymbol{v}\right)^{\mathrm{T}}, \gamma \boldsymbol{I}_{\mathrm{s}}\right)_{\Gamma}, & & \text { for all } \boldsymbol{v}, \\
(q, \nabla \cdot \boldsymbol{u}) & =0, & & \text { for all } q,
\end{aligned}
$$

all at time $t^{n+1}$.

Finally, the boundary position is corrected, where the movement of the boundary is Lagrangian based according to Eq. (6), using a backward Euler scheme

$$
\frac{\boldsymbol{x}_{\Gamma}^{n+1}-\boldsymbol{x}_{\Gamma}^{n}}{\Delta t}=\boldsymbol{u}^{n+1}
$$

for the first time step, and a second-order backward differencing scheme

$$
\frac{\frac{3}{2} \boldsymbol{x}_{\Gamma}^{n+1}-2 \boldsymbol{x}_{\Gamma}^{n}+\frac{1}{2} \boldsymbol{x}_{\Gamma}^{n-1}}{\Delta t}=\boldsymbol{u}^{n+1},
$$

for all subsequent time steps.

\subsection{Finding distance to boundary}

To take the source term into account, we need to find the contribution in each integration point. The term $\exp \left(-\alpha\left(y_{\Gamma}-y\right)\right)$ depends on the position of the boundary $\Gamma$, with $y_{\Gamma} \geq 0$. We find the position of the boundary by performing a Newton-Raphson iteration scheme

$$
\xi^{n+1}=\xi^{n}-\frac{f}{\partial f / \partial \xi}
$$

with $f=\sum_{k}\left(\varphi^{k}(\xi) x_{\Gamma}^{k}\right)-x_{\text {bulk }}$, until $\xi^{n+1}-\xi^{n}<10^{-6}$. Herein, $n$ is the iteration step, $\xi$ is the reference coordinate of the reference line element, and $\varphi(\xi)$ is the shape function. Once the position of the boundary is known, the distance $\left(y_{\Gamma}-y\right)$ can be calculated.

\subsection{Remeshing and projection}

We use Gmsh [31], an open source mesh generator, to generate the mesh. The accuracy of the solution is preserved by remeshing the bulk mesh if the elements are too deformed 
Table 2: Mesh resolution of different surface meshes in the convergence study.

\begin{tabular}{llll}
\hline Mesh & $h_{\text {coarse }}$ & $h_{\text {fine }}$ & Number of nodes \\
\hline M1 & $1.05 R$ & $0.015 R$ & 1121 \\
M2 & $0.7 R$ & $0.01 R$ & 1517 \\
M3 & $0.35 R$ & $0.005 R$ & 3821 \\
M4 & $0.175 R$ & $0.0025 R$ & 11457 \\
M5* & $0.05833 R$ & $0.000833 R$ & 93713 \\
\hline
\end{tabular}

Table 3: Material parameters of PA12 for SLS.

\begin{tabular}{lllll}
\hline Parameter & Symbol & Value & Notes & References \\
\hline Density & $\rho$ & $840 \mathrm{~kg} / \mathrm{m}^{3}$ & $1 \mathrm{bar}, 270^{\circ} \mathrm{C}$, melt & {$[28]$} \\
Surface tension & $\gamma$ & $25 \mathrm{mN} / \mathrm{m}$ & $265^{\circ} \mathrm{C}$, melt & {$[28]$} \\
Melt temperature & $T_{\mathrm{m}}$ & $187^{\circ} \mathrm{C}$ & equilibrium melting point & {$[28]$} \\
Specific heat capacity & $c_{\mathrm{p}}$ & $2.6 \mathrm{~kJ} /(\mathrm{kg} \cdot \mathrm{K})$ & $170^{\circ} \mathrm{C}$ & {$[34]$} \\
Thermal conductivity & $k$ & $0.24 \mathrm{~W} /(\mathrm{m} \cdot \mathrm{K})$ & $170^{\circ} \mathrm{C}$ & {$[34]$} \\
Surface reflectivity & $\zeta_{\mathrm{R}}$ & 0.05 & bulk, $l=200 \mu \mathrm{m}$ & {$[35]$} \\
Transmittance & $\zeta_{\mathrm{T}}$ & 0.20 & bulk, $l=200 \mu \mathrm{m}$ & {$[35]$} \\
Convection coefficient & $h$ & $2.4 \mathrm{~W} /\left(\mathrm{m}^{2} \cdot \mathrm{K}\right)$ & powder surface & {$[36]$} \\
Surface emissivity & $\varepsilon$ & 0.95 & powder surface & {$[36]$} \\
Particle radius & $R$ & $30 \mu \mathrm{m}$ & PA2200 & {$[27]$} \\
Activation energy & $E_{\mathrm{a}}$ & $50 \mathrm{~kJ} / \mathrm{mol}^{2}$ & PA2200 & {$[27]$} \\
Arrhenius coefficient & $\mu_{0}$ & $2 \mathrm{mPa} \cdot \mathrm{s}$ & PA2200 & {$[27]$} \\
Absorption coefficient & $\alpha$ & $7.8 \mathrm{~mm}{ }^{-1}$ & $\zeta_{\mathrm{T}}=\left(1-\zeta_{\mathrm{R}}\right) \exp (-\alpha l)$ & \\
\hline
\end{tabular}

due to large deformations of the domain following [26]. The coordinates of the boundary nodes are retained in this process.

After remeshing, the coordinates $\boldsymbol{x}^{\mathrm{n}}$ and $\boldsymbol{x}^{\mathrm{n}-1}$, and fields $\boldsymbol{u}^{\mathrm{n}}, \boldsymbol{u}^{\mathrm{n}-1}, T^{\mathrm{n}}$, and $T^{\mathrm{n}-1}$ are needed on the new mesh. Therefore, a projection problem is solved, as described in $[26,32]$.

\section{Validation}

\subsection{Isothermal flow}

For an isothermal system, the model is validated using the analytical solution derived by Hopper [33]. This validation is published in [26].

\subsection{Mesh convergence}

To study mesh convergence of the temperature-dependent system, we performed simulations on five different meshes as given in Table 2 , where $h_{\text {coarse }}$ and $h_{\text {fine }}$ are a measure for the maximum and minimum element sizes in the domain, both defined with respect to the radius of the particles. For the convergence study, we disabled the remeshing function of the numerical model. The mesh $\mathrm{M}^{*}$ is used to compute the reference solution. The material and process parameters given in Tables 3 and 4 are used.

First, we performed simulations up to $t=1 \cdot 10^{-4} \mathrm{~s}$, with a time step of $\Delta t=1 \cdot 10^{-7} \mathrm{~s}$. In this time, the laser beam heats up the system. The relative $L^{2}$-error of the temperature 
Table 4: Process and other parameters for SLS.

\begin{tabular}{llll}
\hline Parameter & Symbol & Value & Notes \\
\hline Ambient temperature & $T_{\infty}$ & $170^{\circ} \mathrm{C}$ & \\
Laser power & $P$ & $25 \mathrm{~kW} / \mathrm{m}$ & \\
Beam width & $W$ & $125 \mu \mathrm{m}$ & \\
Laser speed & $u_{\mathrm{b}}$ & $1.25 \mathrm{~m} / \mathrm{s}$ & $t_{\mathrm{b}}=W / u_{\mathrm{b}}$ \\
Heating time & $t_{\mathrm{b}}$ & $1 \cdot 10^{-4} \mathrm{~s}$ & \\
Beam position & $x_{\mathrm{b}}$ & 0 & \\
Stefan-Boltzmann constant & $\sigma_{\mathrm{B}}$ & $5.67 \cdot 10^{-8} \mathrm{~W} /\left(\mathrm{m}^{2} \cdot \mathrm{K}^{4}\right)$ & \\
Gas constant & $G$ & $8.31 \mathrm{~J} /(\mathrm{mol} \cdot \mathrm{K})$ & \\
\hline
\end{tabular}

is determined at $t=1 \cdot 10^{-4} \mathrm{~s}$ at line $y=5 \mu \mathrm{m}, \Gamma_{\varepsilon}$, and is given by

$$
\varepsilon_{T}=\frac{\left(\int_{\Gamma_{\varepsilon}}\left(T^{h}-T^{*}\right)^{2}\right)^{1 / 2}}{\left(\int_{\Gamma_{\varepsilon}} T^{* 2}\right)^{1 / 2}},
$$

with $T^{h}$ the solution of one of the meshes M1 to M4 given in Table 2 and $T^{*}$ the reference solution, computed on M5*. Eq. (38) is evaluated by dividing the line $\Gamma_{\varepsilon}$ into 100 intervals and using a mid-point rule on each interval. The convergence plot is shown in Figure 4.

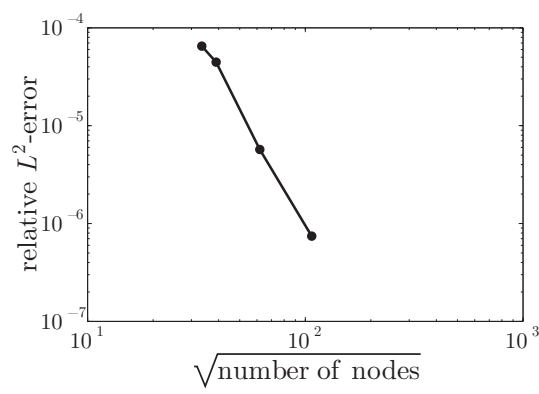

Figure 4: Mesh convergence for the temperature $T$ at $t=1 \cdot 10^{-4} \mathrm{~s}$, using $\Delta t=1 \cdot 10^{-7} \mathrm{~s}$.

Next, we used the situation at $t=1 \cdot 10^{-4} \mathrm{~s}$ as a starting point, to study mesh convergence during the non-linear cooling down of the system. We performed simulations for $t=1 \cdot 10^{-1} \mathrm{~s}$, with a time step of $\Delta t=1 \cdot 10^{-5} \mathrm{~s}$. Again, the relative $L^{2}$-error of the temperature is determined according to Eq. (38). The convergence plot is shown in Figure 5 .

Both Figures 4 and 5 show mesh convergence.

\subsection{Time convergence}

To study time convergence of the temperature-dependent system, we performed simulations using different time steps from $\Delta t=1 \cdot 10^{-4} \mathrm{~s}$ to $\Delta t=1 \cdot 10^{-7} \mathrm{~s}$. The mesh M2, as given in Table 2, is used. The relative $L^{2}$-errors of the temperature are calculated using Eq. (38), with $T^{h}$ the solution of one of the time steps $\Delta t=1 \cdot 10^{-4} \mathrm{~s}$ to $\Delta t=1 \cdot 10^{-7} \mathrm{~s}$ and $T^{*}$ the reference solution, computed using time step $\Delta t=1 \cdot 10^{-8} \mathrm{~s}$. For the convergence study, we disabled the remeshing function of the numerical model.

First, we performed simulations up to $t=1 \cdot 10^{-4} \mathrm{~s}$. The relative $L^{2}$-errors of the temperature are calculated and the convergence plot is shown in Figure 6. 


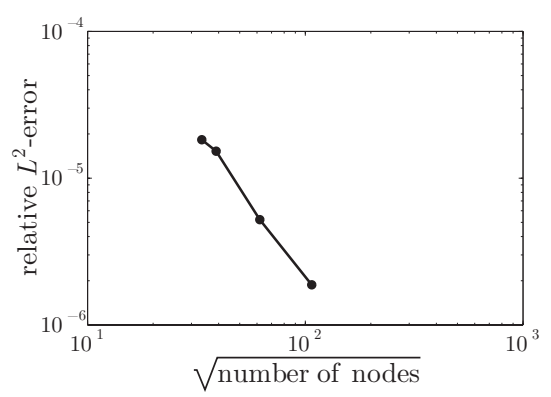

Figure 5: Mesh convergence for the temperature $T$ after $t=1 \cdot 10^{-1} \mathrm{~s}$, using $\Delta t=1 \cdot 10^{-5} \mathrm{~s}$, with starting point the situation at the end of the startup hase.

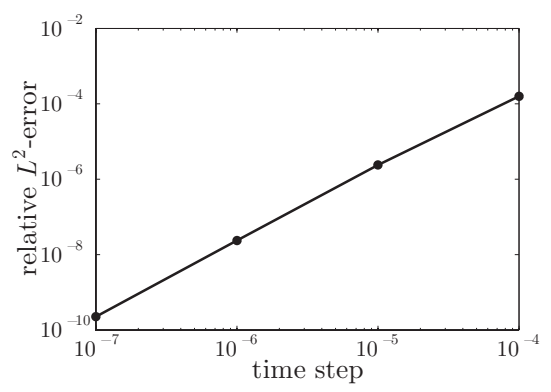

Figure 6: Time convergence for the temperature $T$ at $t=1 \cdot 10^{-4} \mathrm{~s}$, using mesh M2.

Next, we used the situation at $t=1 \cdot 10^{-4} \mathrm{~s}$, with the time step of $\Delta t=1 \cdot 10^{-8} \mathrm{~s}$ as a starting point, to study time convergence during the non-linear cooling down of the system. We performed simulations for $t=1 \cdot 10^{-1} \mathrm{~s}$. The relative $L^{2}$-error of the temperature is calculated and the convergence plot is shown in Figure 7.

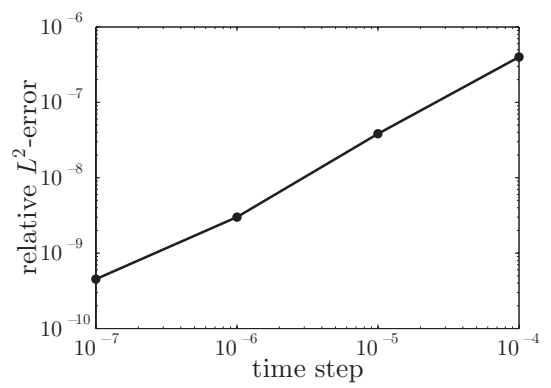

Figure 7: Time convergence for the temperature $T$ after $t=1 \cdot 10^{-1} \mathrm{~s}$, using mesh $M 2$, with starting point the situation at the end of the startup phase using $\Delta t=1 \cdot 10^{-8} \mathrm{~s}$.

Both Figures 6 and 7 show time convergence.

\section{Results}

\subsection{Dimensionless parameters}

As mentioned before, polyamide 12 (PA12) is the polymer that is used most often in SLS. The relevant material parameters of PA12 in the SLS process are given in Table 3. 
Process and other parameters that play a role in SLS are given in Table 4.

\subsubsection{Effective laser power}

To find the characteristic temperature difference, we need to calculate $f_{\mathrm{e}}$, the fraction of the total laser power $P$ that is effectively stored inside the domain. The energy needed to increase the temperature of two circular particles, that are just touching each other, by a certain $\Delta T$, neglecting effects of shear heating and heat loss, is

$$
C=2 A \rho c_{\mathrm{p}} \Delta T,
$$

with $C$ the stored heat per unit length, and $A=\pi R^{2}$ the cross section of one particle. This has to be generated by the laser

$$
C=P f_{\mathrm{e}} t_{\mathrm{b}}
$$

where fraction $f_{\mathrm{e}}$ consists of two parts $f_{\mathrm{e}}=\left(1-\zeta_{\mathrm{R}}\right) f_{\mathrm{a}}$, with $f_{\mathrm{a}}$ the fraction of the beam that is absorbed inside the system, see Figure 8.

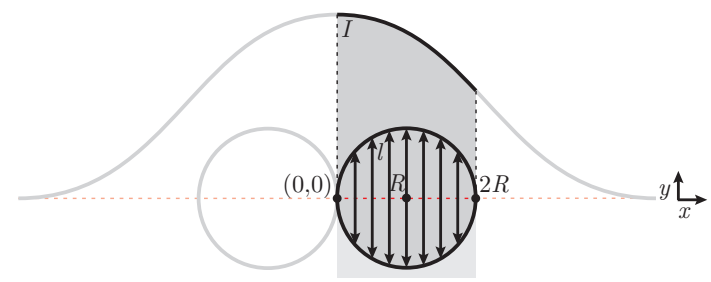

Figure 8: Schematic illustration of $f_{\mathrm{a}}$.

We can calculate the amount of laser power that is absorbed inside the system by taking the difference between the laser intensity that reaches the top of the particles and the laser intensity that exits at the bottom of the particles, and integrating this expression along $x$ :

$$
f_{\mathrm{a}}=2 \frac{1}{\sqrt{\pi W^{2} / 2}} \int_{0}^{2 R} I(x)(1-\exp (-\alpha l(x))) \mathrm{d} x .
$$

Using the values for the particle radius $R$ and beam width $W$ as given in Tables 3 and 4 , the equation for $I(x)$ according to Eq. (11), with $I_{0}=1 \mathrm{~W} / \mathrm{m}^{2}$, and $l(x)=2 \sqrt{R^{2}-(x-R)^{2}}$ the height of the particle at a certain $x$-coordinate, we find $f_{\mathrm{a}}=0.2026$. This means that $20.26 \%$ of the beam energy that reaches the particle is absorbed.

Combining the two contributions gives $f_{\mathrm{e}}=\left(1-\zeta_{\mathrm{R}}\right) f_{\mathrm{a}}=0.1925$.

\subsubsection{Dimensionless groups}

While deriving the dimensionless equations, we found a set of dimensionless groups. Using the data from Tables 3 to 5 , the values of these groups can be calculated. The results are given in Table 6.

The three dimensionless groups in the energy balance of Eq. (19), i.e., the Péclet number Pe, Brinkman number $\mathrm{Br}$, and $\mathrm{N}_{1}$, show respectively that the rate of heat conduction is larger than the rate of heat advection, that the heat produced by viscous dissipation is negligible, and that the source term is dominant. The two dimensionless groups in the definition of the source term of Eq. (20), i.e., $\mathrm{N}_{2}$ and $\mathrm{N}_{3}$, show that the length scale of the system is smaller than both the length scale of the laser beam and the penetration depth. The two dimensionless numbers in the convection-radiation boundary condition of Eq. (22), i.e., the Nusselt number $\mathrm{Nu}$ and $\mathrm{N}_{4}$, show that the convective heat transport is smaller than the radiative heat transport. 
Table 5: Characteristic parameters.

\begin{tabular}{llll}
\hline Parameter & Symbol & Value & Notes \\
\hline Characteristic temperature difference & $\Delta T_{\mathrm{c}}$ & $39.0^{\circ} \mathrm{C}$ & $P f_{\mathrm{e}} t_{\mathrm{b}} /\left(2 A \rho c_{\mathrm{p}}\right)$ \\
Characteristic temperature & $T_{\mathrm{c}}$ & $209.0^{\circ} \mathrm{C}$ & $T_{\infty}+\Delta T_{\mathrm{c}}$ \\
Characteristic viscosity & $\mu_{\mathrm{c}}$ & $528.1 \mathrm{~Pa} \cdot \mathrm{s}$ & $\mu_{0} \exp \left(E_{\mathrm{a}} /\left(G T_{\mathrm{c}}\right)\right)\left(T_{\mathrm{c}}\right.$ in Kelvin $)$ \\
\hline
\end{tabular}

Table 6: Dimensionless groups.

\begin{tabular}{lll}
\hline Number & Value & Notes \\
\hline $\mathrm{Pe}$ & 0.013 & $\rho c_{\mathrm{p}} \gamma R /\left(\mu_{\mathrm{c}} k\right)$ \\
$\mathrm{Br}$ & $1.27 \cdot 10^{-7}$ & $\gamma^{2} /\left(\mu_{\mathrm{c}} k \Delta T_{\mathrm{c}}\right)$ \\
$\mathrm{N}_{1}$ & 113.8 & $R^{2}\left(1-\zeta_{\mathrm{R}}\right) \alpha P /\left(k \Delta T_{\mathrm{c}} \sqrt{\pi W^{2} / 2}\right)$ \\
$\mathrm{N}_{2}$ & 0.058 & $R^{2} / W^{2}$ \\
$\mathrm{~N}_{3}$ & 0.234 & $R \alpha$ \\
$\mathrm{Nu}$ & $3 \cdot 10^{-4}$ & $h R / k$ \\
$\mathrm{~N}_{4}$ & 0.002 & $R \sigma_{\mathrm{B}} \varepsilon 4 T_{\infty}^{3} / k$ \\
\hline
\end{tabular}

\subsection{Characteristic temperature difference}

As given in Table 5, we found analytically that the characteristic temperature difference is $39.0^{\circ} \mathrm{C}$. This characteristic temperature difference should approximate the temperature difference calculated using the computational model. We performed simulations using the parameters given in Tables 3 and 4 without shear heating and heat loss to the environment. From the computation, we found a value of $T=208.8^{\circ} \mathrm{C}$ for the average temperature in the domain, leading to a temperature difference of $\Delta T=38.8^{\circ} \mathrm{C}$. This shows that the results of the temperature difference from the numerical simulation and the analytical calculation, using the approximation for the effective laser power and particle area, are in good agreement.

\subsection{Effect of parameters}

We split the total sintering process into three phases, i.e., the startup phase, the diffusion phase, and the complete flow phase. In each of these phases, a different timescale dominates the process. In the startup phase, from $t=0$ to $t=1 \cdot 10^{-4} \mathrm{~s}$, the laser beam heats up the system. In this short time, the temperature of the system increases significantly. The diffusion phase is chosen to be up to $t=1 \cdot 10^{-2} \mathrm{~s}$, based on the diffusion time $t_{\text {diff }}=R^{2} \rho c_{\mathrm{p}} / k=0.0082 \mathrm{~s}$. During the diffusion phase, the temperature difference between the minimum and maximum temperature, $T_{\min }$ and $T_{\max }$, inside the system decreases. At the end of the diffusion phase, the minimum and maximum temperature should be approximately equal, and we call this temperature the equilibrium temperature $T_{\text {eq }}$. In the complete flow phase, the system is monitored until the maximum temperature in the system reaches the melting temperature $T_{\max }=T_{\mathrm{m}}=187^{\circ} \mathrm{C}$, the material solidifies, and the viscosity goes to infinity $\mu(T) \rightarrow \infty$. In all simulations, the values of Tables 3 and 4 are used, unless stated differently. For each of these phases, we changed six input parameters separately: the parameters used in the Arrhenius relation for the viscosity $E_{\mathrm{a}}$ and $\mu_{0}$, the laser power $P$, the beam width $W$, the ambient temperature $T_{\infty}$, the position of the beam $x_{\mathrm{b}}$, and the convection coefficient $h$. In the following sections, we discuss the most important observations. 


\subsubsection{Viscosity}

To change the temperature-dependent viscosity, we varied the activation energy $E_{\mathrm{a}}$ and the Arrhenius coefficient $\mu_{0}$. In this way, all parameters concerning the energy balance and the associated boundary conditions are fixed, except for the temperature-dependent viscosity playing a role in the advection term and the viscous dissipation term. We used the values for PA2200 as given in Table 3 , and the values $E_{\mathrm{a}}=70 \cdot 10^{3} \mathrm{~J} / \mathrm{mol}$ and $\mu_{0}=1.4 \cdot 10^{-4} \mathrm{~Pa} \cdot \mathrm{s}$ for Orgasol [27]. The behavior of the temperature-dependent viscosity is given in Figure 9. During the startup phase, we monitor the maximum temperature $T_{\max }$ in the system. In the diffusion phase, we look at both the minimum and maximum temperature, $T_{\min }$ and $T_{\max }$. For the complete flow phase, we plot the evolution of the contact line $L$ in time, and the maximum temperature $T_{\max }$ inside the domain. These results can be found in Figure 10.

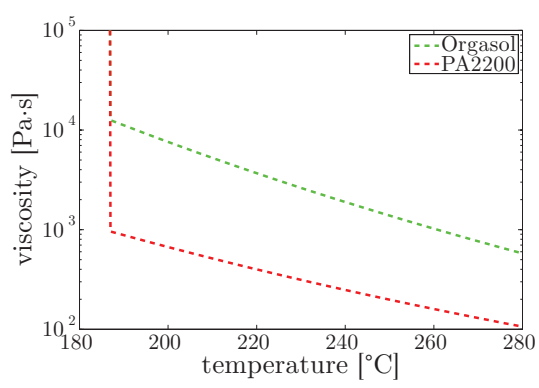

Figure 9: Temperature-dependent viscosity of PA2200 and Orgasol.

(a)

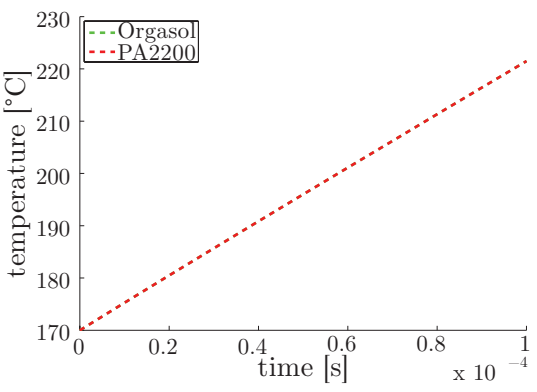

(c)

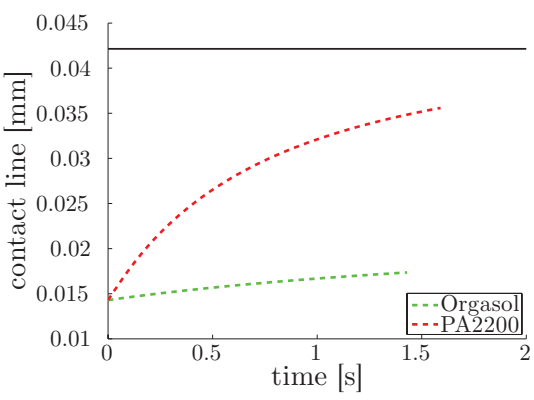

(b)

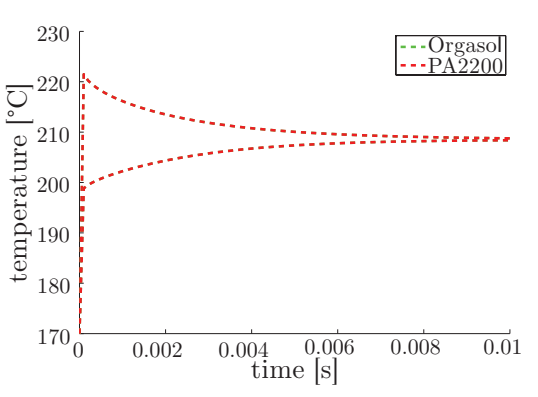

(d)

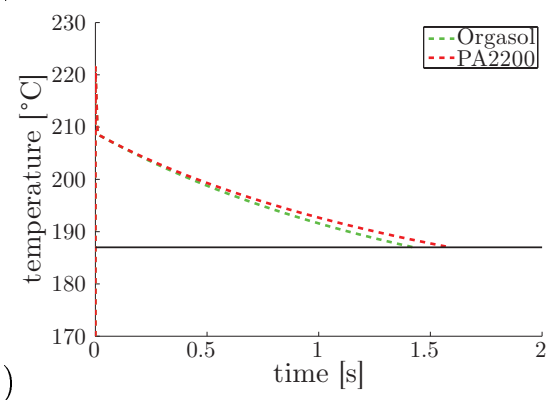

Figure 10: Behavior of the system for different parameters in the Arrhenius relation for the viscosity during (a) the startup phase $\left(T_{\max }\right)$, (b) the diffusion phase $\left(T_{\min }\right.$ and $\left.T_{\max }\right)$, and (c-d) the complete flow phase ( $L$ and $T_{\max }$, respectively). In (c), the solid line denotes the contact line of the full circle. In (d), the solid line shows the melting temperature $T_{\mathrm{m}}$.

During the startup phase and the diffusion phase, the viscous dissipation and effects 
from flow can be neglected. This leads to the same behavior of the temperature $T$ for the two materials in these phases, and therefore overlapping lines in Figures 10(a-b). For the same temperature $T$, the viscosity $\mu(T)$ of PA2200 is lower than that of Orgasol, as shown in Figure 9. Therefore, the increase in contact line $L$ is faster for PA2200, if we look at the complete flow phase. Simultaneously, the length of the boundary decreases, leading to a slightly slower heat loss for PA2200 compared to Orgasol.

In Figure 11, the temperature distribution (a) at the end of the startup phase for both PA2200 and Orgasol, (b) at the end of the diffusion phase for both PA2200 and Orgasol, and at the end of the complete flow phase for (c) PA2200 and (d) Orgasol are shown. At the end of the startup phase, the maximum temperatures $T_{\max }$ can be found at the boundary of the particles slightly left and right of the contact region at the top half. At the end of the diffusion phase, the maximum temperature $T_{\max }$ is found in the contact region $x=0$ of the top half, just below the boundary. At the end of the complete flow phase, the maximum temperature $T_{\max }$ lies in the center of the system $(x, y)=(0,0)$ for both PA2200 and Orgasol.

Summarizing the results in this section, fast fusion can be achieved if the viscosity $\mu(T)$ is low just above the melting temperature.

(a)
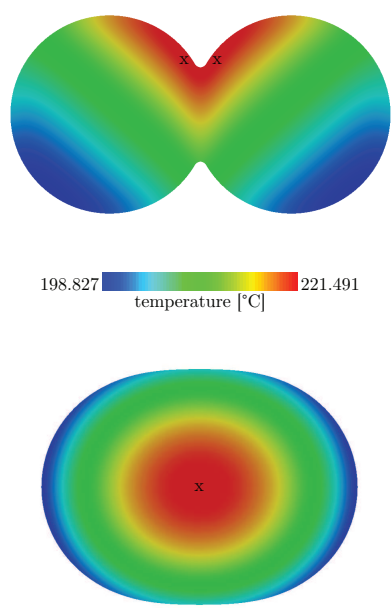

(c)

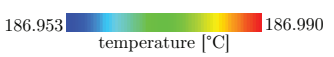

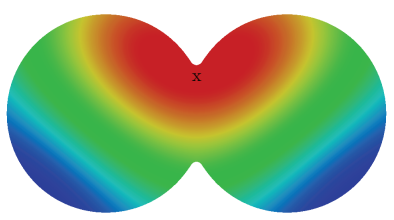

(b)
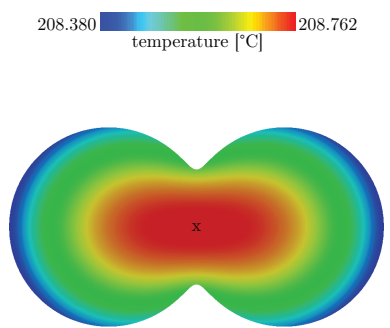

(d)

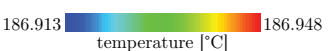

Figure 11: Temperature distribution at (a) the end of the startup phase for both PA2200 and Orgasol, (b) the end of the diffusion phase for both PA2200 and Orgasol, (c) the end of the complete flow phase for PA2200 $(t=1.59 \mathrm{~s})$, and $(\mathrm{d})$ at the end of the complete flow phase for Orgasol $(t=1.43 \mathrm{~s})$. Note that the ranges of the color bars are different for each subfigure, to emphasize the temperature distribution for each phase. The ' $x$ ' marks the location of the maximum temperature $T_{\max }$.

\subsubsection{Laser power}

We varied the laser power using $P=12.5 \mathrm{~kW} / \mathrm{m}, P=25 \mathrm{~kW} / \mathrm{m}$, and $P=50 \mathrm{~kW} / \mathrm{m}$, with $P=25 \mathrm{~kW} / \mathrm{m}$ the basic value as given in Table 4 . During the startup phase, we monitor the maximum temperature $T_{\max }$ in the system. In the diffusion phase, we look at both the minimum and maximum temperature, $T_{\min }$ and $T_{\max }$. For the complete flow phase, we plot the evolution of the contact line $L$ in time, and the maximum temperature $T_{\max }$ inside the domain. These results are shown in Figure 12. 


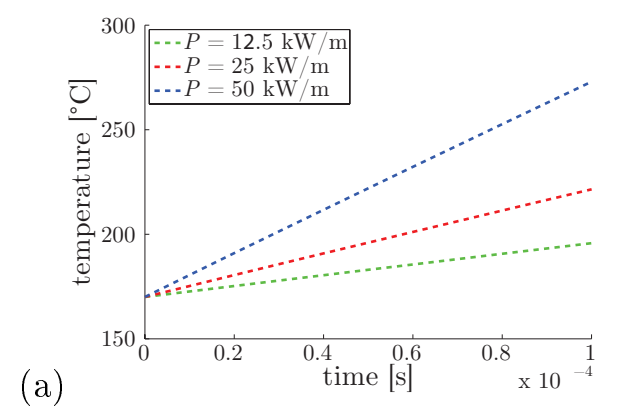

(a)

(c)

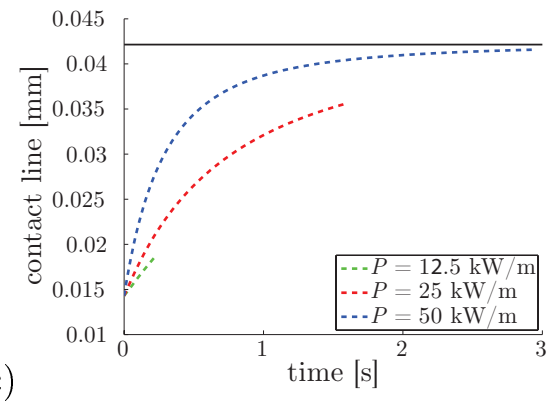

(b)

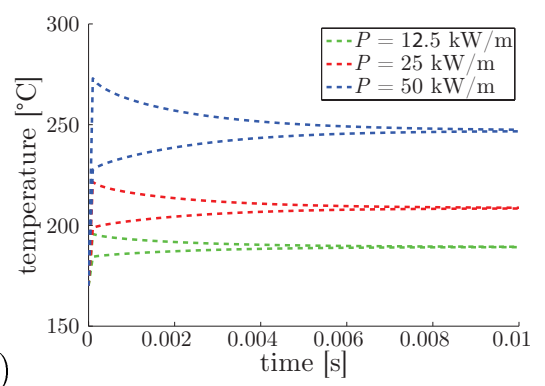

(d)

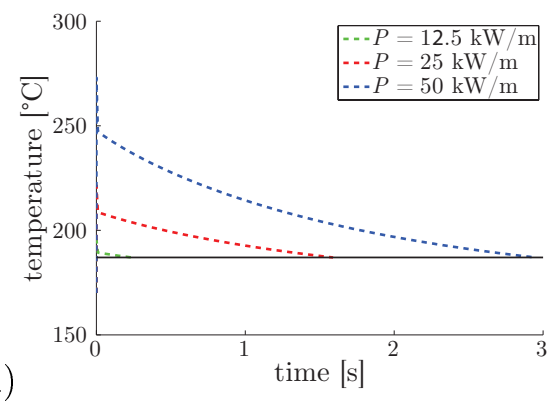

Figure 12: Behavior of the system for different values of the laser power during (a) the startup phase $\left(T_{\max }\right),(\mathrm{b})$ the diffusion phase $\left(T_{\min }\right.$ and $\left.T_{\max }\right)$, and (c-d) the complete flow phase $(L$ and $T_{\max }$, respectively). In (c), the solid line denotes the contact line of the full circle. In (d), the solid line shows the melting temperature $T_{\mathrm{m}}$.

The maximum temperature $T_{\max }$ increases linear in time during the startup phase for every value of the laser power $P$. Besides, the change in maximum temperature $\Delta T_{\max }=T_{\max }-T_{\infty}$ scales linearly with the laser power $P$, i.e., if the laser power is twice as high, the change in maximum temperature $\Delta T_{\max }$ doubles. The same linear relation is found for the change in equilibrium temperature $\Delta T_{\mathrm{eq}}=T_{\mathrm{eq}}-T_{\infty}$ at the end of the diffusion phase. With increasing laser power $P$, the equilibrium temperature $T_{\text {eq }}$ of the system increases, and the viscosity $\mu(T)$ decreases, leading to a faster increase in contact line $L$. For laser power $P=50 \mathrm{~kW} / \mathrm{m}$, the two particles become almost circular.

The results in this section indicate that increasing the laser power $P$ is beneficial for the flowability of the system, but increases the process time.

\subsubsection{Beam width}

We changed the beam width using $W=30 \mu \mathrm{m}, W=125 \mu \mathrm{m}$, and $W=300 \mu \mathrm{m}$, with $W=125 \mu \mathrm{m}$ the basic value as given in Table 4 . During the startup phase, we monitor the maximum temperature $T_{\max }$ in the system. In the diffusion phase, we look at both the minimum and maximum temperature, $T_{\min }$ and $T_{\max }$. For the complete flow phase, we plot the evolution of the contact line $L$ in time, and the maximum temperature $T_{\max }$ inside the domain. These results can be found in Figure 13.

If the beam width $W$ is smaller, less energy is wasted outside the domain, and the energy is provided more locally. This leads to an increase in both the maximum temperature $T_{\max }$ and the temperature difference $\Delta T_{\Omega}=T_{\max }-T_{\min }$ inside the domain. The increase in maximum temperature $T_{\max }$ during the startup phase is linear. For smaller beam width $W$, the equilibrium temperature $T_{\text {eq }}$ of the system is higher, resulting in a lower viscosity $\mu(T)$. This leads to a faster increase in contact line $L$, and a longer time for the maximum temperature $T_{\max }$ to reach the melting temperature $T_{\mathrm{m}}$ in the complete flow phase. Note that the chosen beam width $W=30 \mu \mathrm{m}$ in combination with the other 


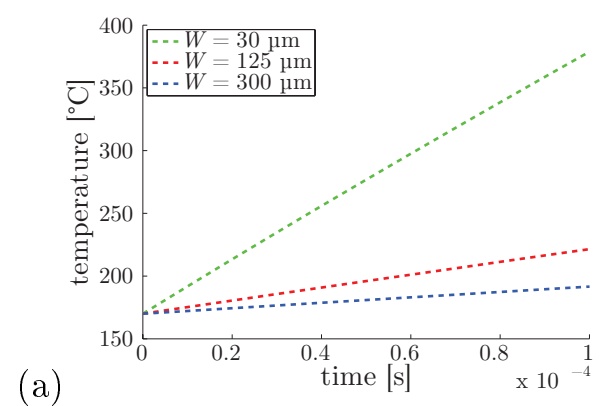

(a)

(c)

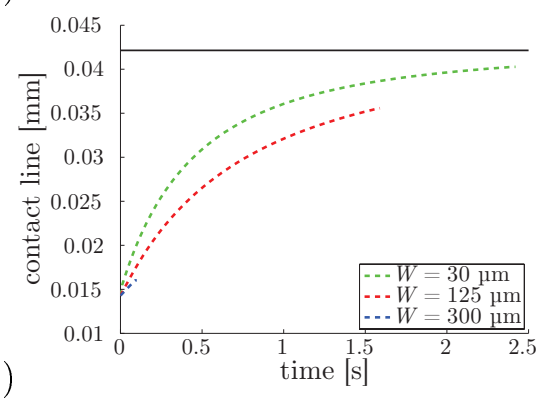

(b)

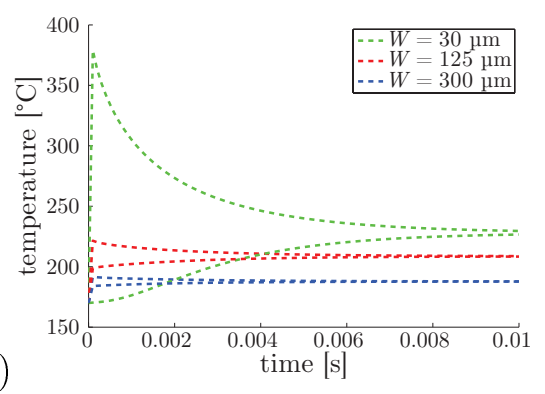

(d)

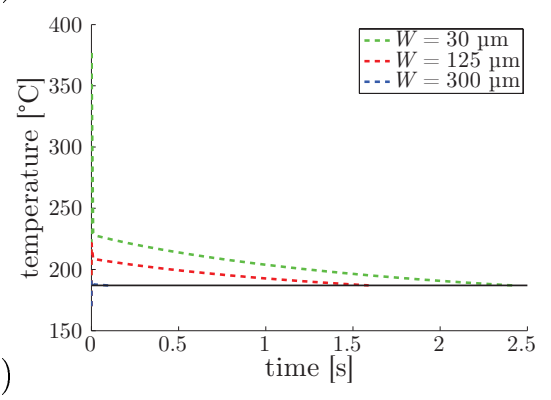

Figure 13: Behavior of the system for different values of the beam width during (a) the startup phase $\left(T_{\max }\right),(\mathrm{b})$ the diffusion phase $\left(T_{\min }\right.$ and $\left.T_{\max }\right)$, and $(\mathrm{c}-\mathrm{d})$ the complete flow phase $(L$ and $T_{\max }$, respectively). In (c), the solid line denotes the contact line of the full circle. In (d), the solid line shows the melting temperature $T_{\mathrm{m}}$.

process parameters may reach temperatures that will thermally degrade or ablate the polymer.

In Figure 14, the temperature distribution at the end of the startup phase, at the end of the diffusion phase, and at the end of the complete flow phase for $W=30 \mu \mathrm{m}$ and $W=300 \mu \mathrm{m}$ is shown. For $W=30 \mu \mathrm{m}$, the maximum temperature $T_{\max }$ can be found at the boundary of the system in the contact region $x=0$ of the top half at the end of the startup phase. At the end of the diffusion phase, the maximum temperature $T_{\max }$ is found in the contact region below the boundary at approximately $(x, y)=(0, L / 3)$. For $W=300 \mu \mathrm{m}$, the maximum temperatures $T_{\max }$ can be found at the boundary of the particles at approximately $x=-R / 3$ and $x=R / 3$ of the top half at the end of the startup phase, while at the end of the diffusion phase, the highest temperatures are found just below the boundary at approximately $x=-2 R / 3$ and $x=2 R / 3$ of the top half. At the end of the complete flow phase, the maximum temperature $T_{\max }$ is located at the center $(x, y)=(0,0)$ for both beam widths.

Frome the results in this section, we see that decreasing the beam width $W$ is beneficial for the flowability of the system, but increases the process time.

\subsubsection{Ambient temperature}

We used different values for the ambient temperature, which is also taken as the initial temperature, $T_{\infty}=155^{\circ} \mathrm{C}, T_{\infty}=170^{\circ} \mathrm{C}$, and $T_{\infty}=185^{\circ} \mathrm{C}$, with $T_{\infty}=170^{\circ} \mathrm{C}$ the basic value as given in Table 4. During the startup phase, we monitor the maximum temperature $T_{\max }$ in the system. In the diffusion phase, we look at both the minimum and maximum temperature, $T_{\min }$ and $T_{\max }$. For the complete flow phase, we plot the evolution of the contact line $L$ in time, and the maximum temperature $T_{\max }$ inside the domain. These results are shown in Figure 15.

In the startup phase, the linear increase in maximum temperature $T_{\max }$ follows the 


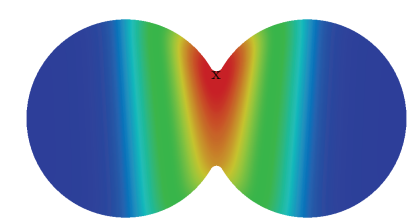

(a)
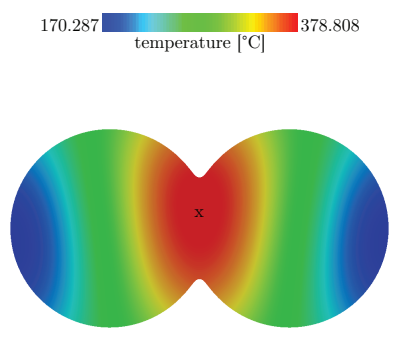

(c)

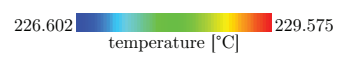

(e)

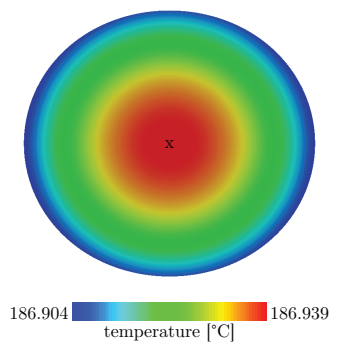

(b)
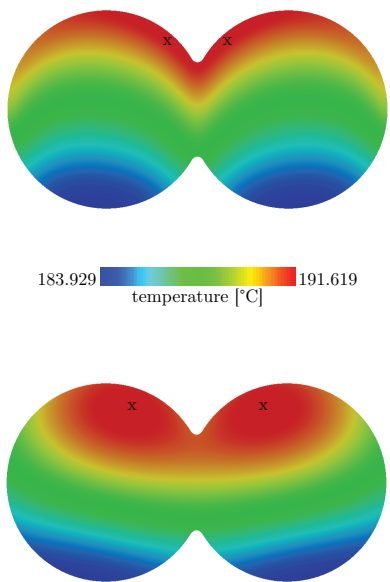

(d)
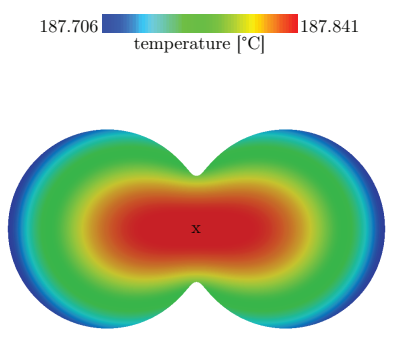

(f)

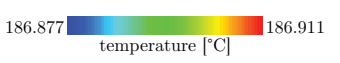

Figure 14: Temperature distribution at (a) the end of the startup phase for $W=30 \mu \mathrm{m}$, (b) the end of the startup phase for $W=300 \mu \mathrm{m}$, (c) the end of the diffusion phase for $W=30 \mu \mathrm{m}$, (d) the end of the diffusion phase for $W=300 \mu \mathrm{m}$, (e) the end of the complete flow phase for $W=30 \mu \mathrm{m}(t=2.42 \mathrm{~s})$, and (f) the end of the complete flow phase for $W=300 \mu \mathrm{m}(t=0.1 \mathrm{~s})$. Note that the ranges of the color bars are different for each subfigure, to emphasize the temperature distribution for each phase. The ' $x$ ' marks the location of the maximum temperature $T_{\max }$.

same slope for the different values of the ambient temperature $T_{\infty}$, however the lines are shifted with respect to the initial temperature $T(t=0)=T_{\infty}$. The behavior of the system during the diffusion phase is identical for the three cases, but again shifted with respect to the initial temperature, i.e., the higher the ambient temperature $T_{\infty}$, and thus the initial temperature, the higher the equilibrium temperature $T_{\mathrm{eq}}$ of the system. For the complete flow phase holds that the higher the equilibrium temperature $T_{\mathrm{eq}}$, the lower the viscosity $\mu(T)$ is, the faster the increase in contact line $L$, and the longer it takes for the maximum temperature $T_{\max }$ to reach the melting temperature $T_{\mathrm{m}}$. For ambient temperature $T_{\infty}=185^{\circ} \mathrm{C}$, the two particles become fully circular during the complete flow phase.

To summarize, a higher ambient temperature $T_{\infty}$ improves the flowability, but significantly decreases the cooling rate, which is undesirable from the processing perspective. 


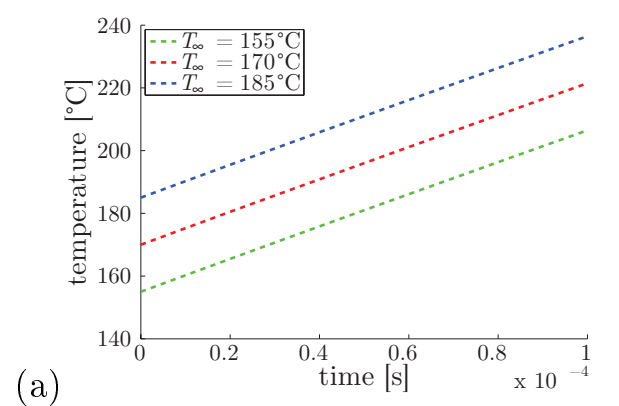

(a)

(c)

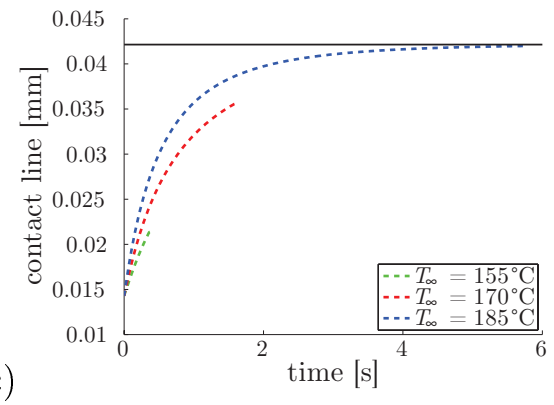

(b)

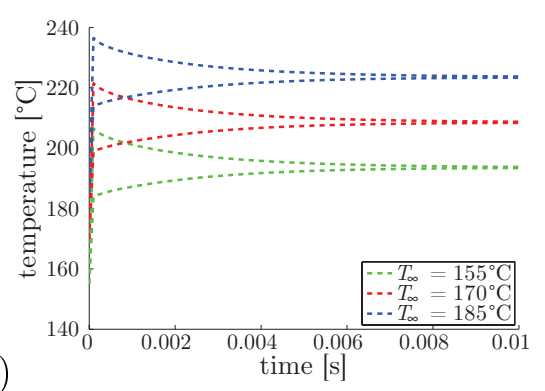

(d)

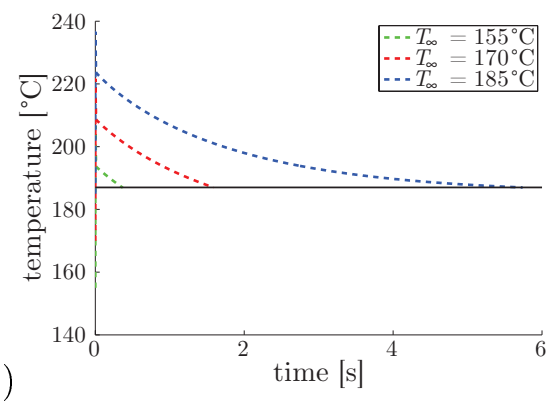

Figure 15: Behavior of the system for different values of the ambient temperature during (a) the startup phase $\left(T_{\max }\right)$, (b) the diffusion phase $\left(T_{\min }\right.$ and $\left.T_{\max }\right)$, and $(\mathrm{c}-\mathrm{d})$ the complete flow phase ( $L$ and $T_{\max }$, respectively). In (c), the solid line denotes the contact line of the full circle. In (d), the solid line shows the melting temperature $T_{\mathrm{m}}$.

\subsubsection{Beam position}

We varied the position of the beam using $x_{\mathrm{b}}=0, x_{\mathrm{b}}=-30 \mu \mathrm{m}$, and $x_{\mathrm{b}}=-60 \mu \mathrm{m}$, with $x_{\mathrm{b}}=0$ the basic value. During the startup phase, we monitor the maximum temperature $T_{\max }$ in the system. In the diffusion phase, we look at both the minimum and maximum temperature, $T_{\min }$ and $T_{\max }$. For the complete flow phase, we plot the evolution of the contact line $L$ in time, and the maximum temperature $T_{\max }$ inside the domain. These results can be found in Figure 16.

During the startup phase, the increase in maximum temperature $T_{\max }$ is linear. The maximum temperature $T_{\max }$ increases slightly more if the laser beam is focused on the thickest part of the particle system, i.e., $x_{\mathrm{b}}=-30 \mu \mathrm{m}$, because the laser has more material to penetrate and heat up. If the beam is focused further away from the center of the system, the difference between the the minimum temperature $T_{\min }$ and maximum temperature $T_{\max }$ increases. Consequently, the time it takes to equilibrate the temperature $T$ during the diffusion phase is longer, due to the higher temperature difference and larger length scales involved. Therefore, the equilibrium temperature $T_{\text {eq }}$ is not reached yet during the diffusion phase for beam positions $x_{\mathrm{b}}=-30 \mu \mathrm{m}$ and $x_{\mathrm{b}}=-60 \mu \mathrm{m}$. Although the maximum temperature $T_{\max }$ is slightly higher for $x_{\mathrm{b}}=-30 \mu \mathrm{m}$, the equilibrium value of the temperature $T_{\text {eq }}$ seems to reach a value that is lower if the beam is focused further away from the center of the system, because the minimum temperature $T_{\min }$ in the domain is lower. For the complete flow phase holds that the closer the beam to $x_{\mathrm{b}}=0$, the higher the maximum temperature $T_{\max }$ of the system, the lower the viscosity $\mu(T)$, the faster the increase in contact line $L$, and the longer it takes for the maximum temperature $T_{\max }$ to reach the melting temperature $T_{\mathrm{m}}$.

In Figure 17, the temperature distribution at the end of the startup phase, at the end of the diffusion phase, and at the end of the complete flow phase for $x_{\mathrm{b}}=-30 \mu \mathrm{m}$ and $x_{\mathrm{b}}=-60 \mu \mathrm{m}$ is shown. For $x_{\mathrm{b}}=-30 \mu \mathrm{m}$, the maximum temperature $T_{\max }$ can 


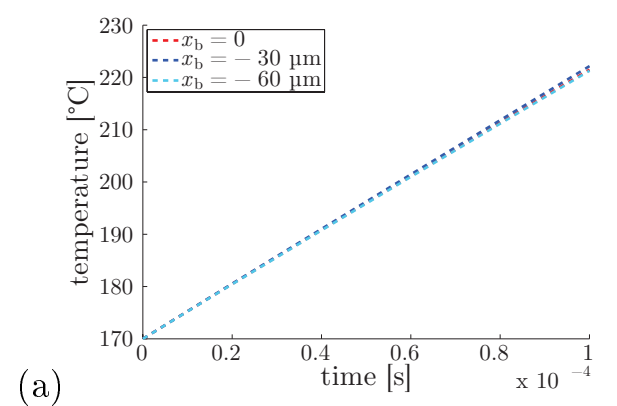

(a)

(c)

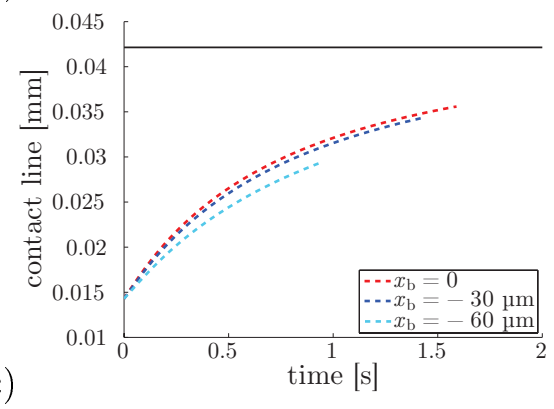

(b)

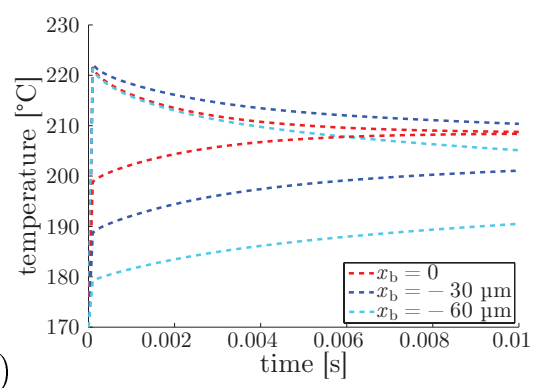

(d)

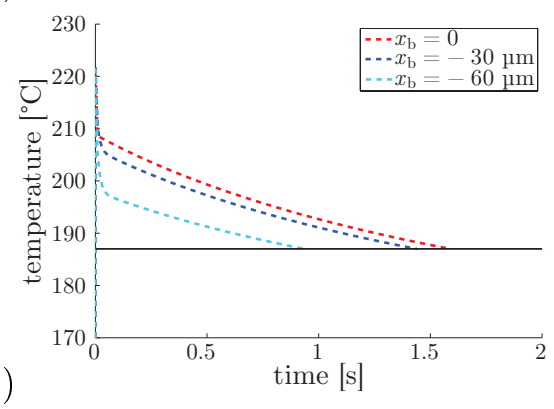

Figure 16: Behavior of the system for different positions of the beam during (a) the startup phase $\left(T_{\max }\right),(\mathrm{b})$ the diffusion phase $\left(T_{\min }\right.$ and $\left.T_{\max }\right)$, and $(\mathrm{c}-\mathrm{d})$ the complete flow phase $(L$ and $T_{\max }$, respectively). In (c), the solid line denotes the contact line of the full circle. In (d), the solid line shows the melting temperature $T_{\mathrm{m}}$.

be found at the boundary at $x=-R$ of the top half at the end of the startup phase. During the diffusion phase, the maximum temperature $T_{\max }$ shifts slightly to the left and is found at the boundary at approximately $(x, y)=(-2 R, L / 2)$ of the top half at the end of the diffusion phase. For $x_{\mathrm{b}}=-60 \mu \mathrm{m}$, the maximum temperature $T_{\max }$ can be found at the boundary of the system at approximately $x=-5 R / 3$ of the top half at the end of the startup phase, and the maximum temperature $T_{\max }$ is found at the boundary at $x=-2 R$ of the top half at the end of the diffusion phase. For both cases, the maximum temperature $T_{\max }$ is located at the center of the system $(x, y)=(0,0)$ at the end of the complete flow phase.

The upshot is that in this model system, the best beam position is at $x_{\mathrm{b}}=0$ for fast fusion. It should be noted that during SLS the beam moves over the powder bed.

\subsubsection{Convective heat transfer coefficient}

The convective heat transfer coefficient, or short 'convection coefficient' depends strongly on the surroundings of the system and is often used to fit numerical simulations to experimental results. Therefore, the chosen value in Table 3 might be low compared to the actual convection coefficient in the SLS process. We varied the convection coefficient using $h=2.4 \mathrm{~W} /(\mathrm{m} \cdot \mathrm{K}), h=24 \mathrm{~W} /(\mathrm{m} \cdot \mathrm{K})$, and $h=240 \mathrm{~W} /(\mathrm{m} \cdot \mathrm{K})$, with $h=2.4 \mathrm{~W} /(\mathrm{m} \cdot \mathrm{K})$ the basic value. For $h=2.4 \mathrm{~W} /(\mathrm{m} \cdot \mathrm{K})$, the loss term is dominated by radiation. For $h=24 \mathrm{~W} /(\mathrm{m} \cdot \mathrm{K})$, the convection and radiation are approximately in equilibrium in the loss term. For $h=240 \mathrm{~W} /(\mathrm{m} \cdot \mathrm{K})$, the loss term is dominated by convection. During the startup phase, we monitor the maximum temperature $T_{\max }$ in the system. In the diffusion phase, we look at both the minimum and maximum temperature, $T_{\min }$ and $T_{\max }$. For the complete flow phase, we plot the evolution of the contact line $L$ in time, and the maximum temperature $T_{\max }$ inside the domain. These results can be found in Figure 18.

During the startup phase, the same behavior of the temperature $T$ can be seen for the 


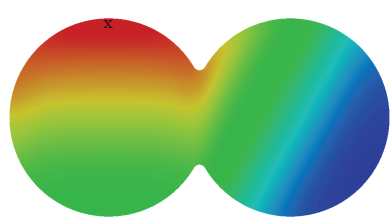

(a)
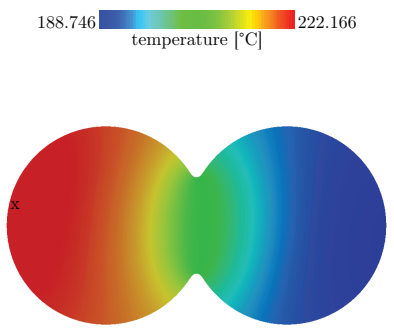

(c)

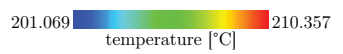

(e)
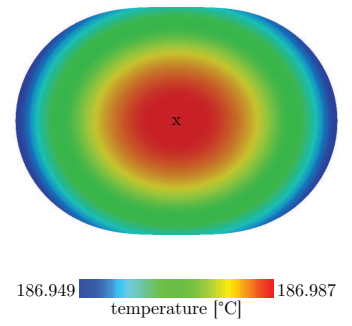

(b)
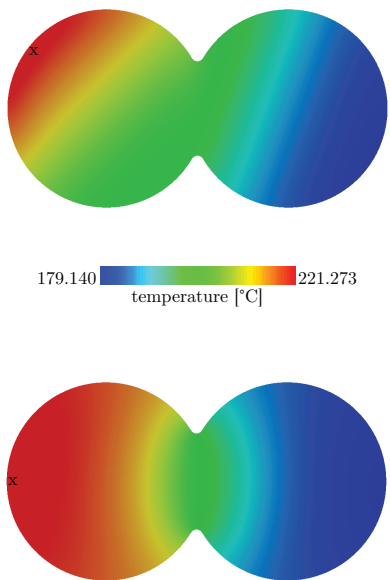

(d)
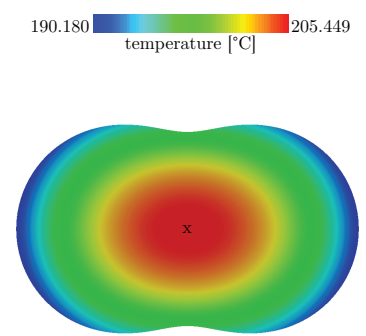

(f)

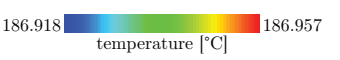

Figure 17: Temperature distribution at (a) the end of the startup phase for $x_{\mathrm{b}}=-30 \mu \mathrm{m}$, (b) the end of the startup phase for $x_{\mathrm{b}}=-60 \mu \mathrm{m}$, (c) the end of the diffusion phase for $x_{\mathrm{b}}=-30 \mu \mathrm{m},(\mathrm{d})$ the end of the diffusion phase for $x_{\mathrm{b}}=-60 \mu \mathrm{m},(\mathrm{e})$ the end of the complete flow phase for $x_{\mathrm{b}}=-30 \mu \mathrm{m}(t=1.44 \mathrm{~s})$, and ( $\left.\mathrm{f}\right)$ the end of the complete flow phase for $x_{\mathrm{b}}=$ $-60 \mu \mathrm{m}(t=0.94 \mathrm{~s})$. Note that the ranges of the color bars are different for each subfigure, to emphasize the temperature distribution for each phase. The ' $x$ ' marks the location of the maximum temperature $T_{\max }$.

three values of the convection coefficient $h$, and therefore overlapping lines in Figure 18(a). This means that the heat transfer through convection is negligible during the startup phase. During the diffusion phase, the first differences in the behavior of the temperature $T$ appear. The temperature $T$ decreases faster and the equilibrium temperature $T_{\text {eq }}$ is lower for higher convection coefficient $h$. For the complete flow phase holds that the temperature $T$ decreases faster for higher convection coefficient $h$, resulting in a higher viscosity $\mu(T)$ a slower increase in contact line $L$, and a shorter time before the maximum temperature $T_{\max }$ has reached the melting temperature $T_{\mathrm{m}}$.

To summarize, a lower convection coefficient $h$ improves the flowability, but significantly decreases the cooling rate, which is undesirable from the processing perspective. The convection coefficient $h$ depends on the complicated process of heat transfer to the surrounding air. The appropriate value can be determined from experiments. 


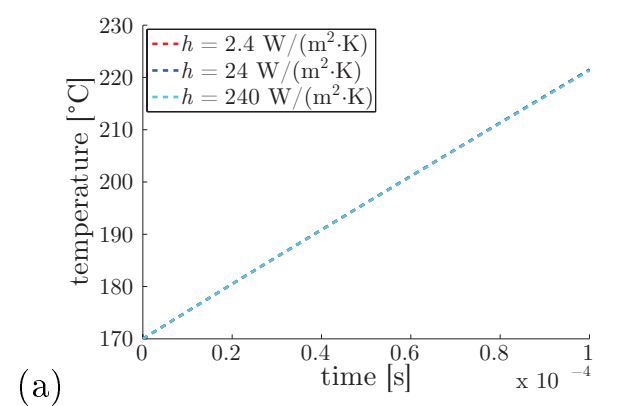

(a)

(c)

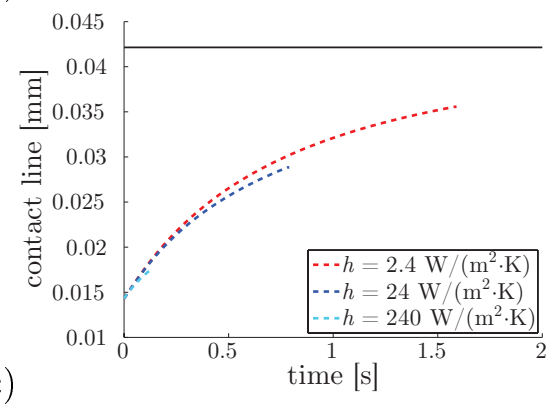

(b)

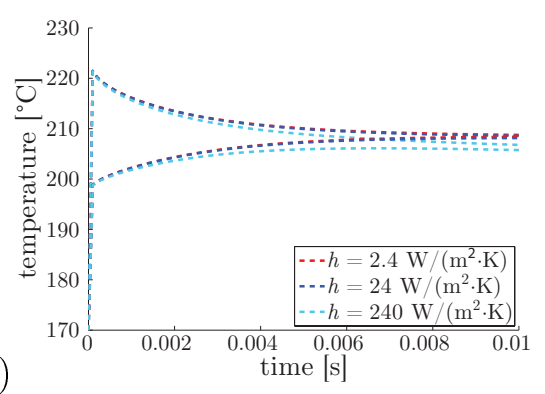

(d)

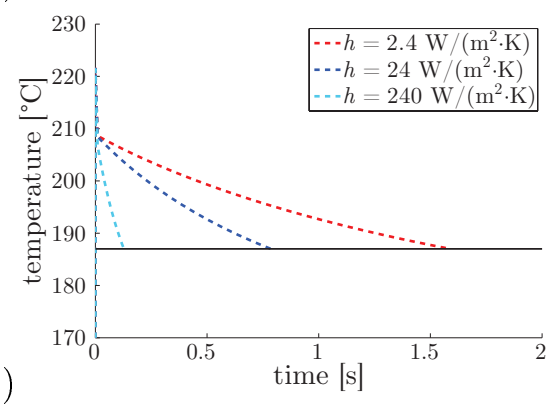

Figure 18: Behavior of the system for different values of the convection coefficient during (a) the startup phase $\left(T_{\max }\right)$, (b) the diffusion phase $\left(T_{\min }\right.$ and $\left.T_{\max }\right)$, and $(\mathrm{c}-\mathrm{d})$ the complete flow phase ( $L$ and $T_{\max }$, respectively). In (c), the solid line denotes the contact line of the full circle. In (d), the solid line shows the melting temperature $T_{\mathrm{m}}$.

\subsection{Moving beam}

During the SLS process, the laser beam scans the polymer powder using a moving laser beam. Therefore, we altered the description of the source term Eq. (15), to take the velocity of the beam into account. This results in

$$
Q(x, y)=\alpha\left(1-\zeta_{\mathrm{R}}\right) \frac{P}{\sqrt{\pi W^{2} / 2}} \exp \left(-2 \frac{\left(x-x_{\mathrm{b}}-u_{\mathrm{b}} t\right)^{2}}{W^{2}}\right) \exp \left(-\alpha\left(y_{\Gamma}-y\right)\right) .
$$

We start at position $x_{\mathrm{b}}=-187.5 \mu \mathrm{m}$, such that the particles are just out of reach for the laser beam. For $t_{\mathrm{b}}=3 \cdot 10^{-4} \mathrm{~s}$, we let the beam move with velocity $u_{\mathrm{b}}=1.25 \mathrm{~m} / \mathrm{s}$, as given in Table 4 . All other used material and process parameters are shown in Tables 3 and 4 .

We compared the results of the moving beam with simulations of a fixed beam at position $x_{\mathrm{b}}=0$ with $P=25 \mathrm{~kW} / \mathrm{m}$ for $t_{\mathrm{b}}=1 \cdot 10^{-4} \mathrm{~s}$ (equal to the standard parameters given in Tables 3 and 4 ), and with $P=11.722 \mathrm{~kW} / \mathrm{m}$ for $t_{\mathrm{b}}=3 \cdot 10^{-4} \mathrm{~s}$. The latter is the laser power $P$ at which the amount of absorbed energy for a static beam is equivalent to that of a moving beam for the same heating time $t_{\mathrm{b}}$.

Again, we looked at the startup phase, now defined from $t=0$ to $t=3 \cdot 10^{-4} \mathrm{~s}$, the diffusion phase, up to $t=1 \cdot 10^{-2} \mathrm{~s}$, and the complete flow phase. During the startup phase, we monitor the maximum temperature $T_{\max }$ in the system. In the diffusion phase, we look at both the minimum and maximum temperature, $T_{\min }$ and $T_{\max }$. For the complete flow phase, we plot the evolution of the contact line $L$ in time, and the maximum temperature $T_{\max }$ inside the domain. These results can be found in Figure 19.

During the startup phase, the increase in maximum temperature $T_{\max }$ for the moving beam has a delay with respect to the fixed beams. Around $t=1.5 \cdot 10^{-4} \mathrm{~s}$, the slope of the graph of the moving beam is equal to the slope of the fixed beam with laser power $P=25 \mathrm{~kW} / \mathrm{m}$. At that moment, the center of the moving laser beam is positioned 


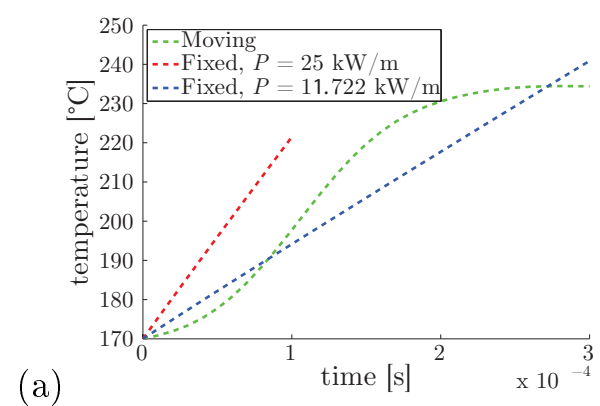

(a)

(c)

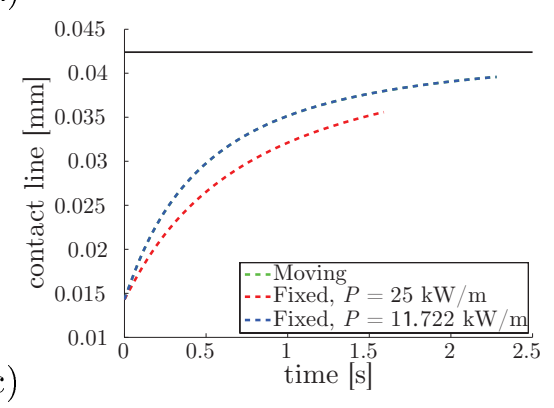

(b)

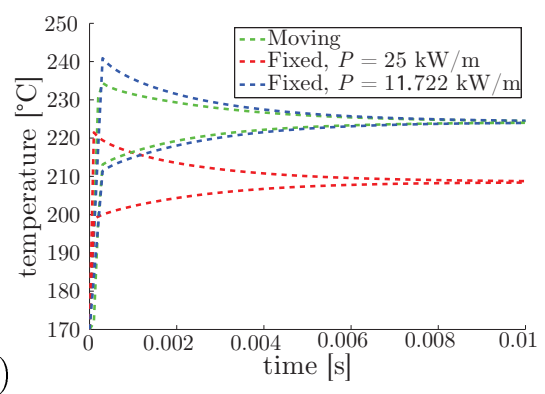

(d)

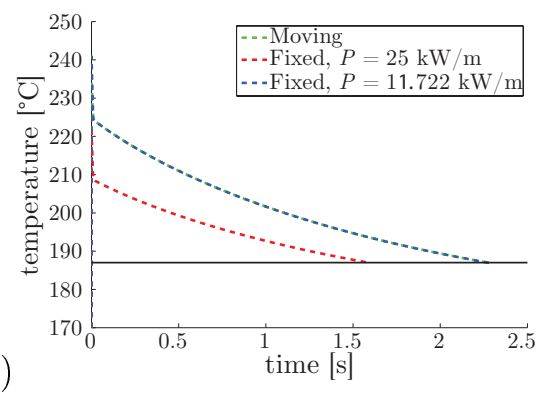

Figure 19: Behavior of the system for a moving beam compared to a fixed beam during (a) the startup phase $\left(T_{\max }\right)$, (b) the diffusion phase $\left(T_{\min }\right.$ and $\left.T_{\max }\right)$, and (c-d) the complete flow phase ( $L$ and $T_{\max }$, respectively). In (c), the solid line denotes the contact line of the full circle. In (d), the solid line shows the melting temperature $T_{\mathrm{m}}$.

close to the origin $(0,0)$, which is equal to the position $x_{\mathrm{b}}=0$ of the fixed laser beam. Since both the position and the prescribed laser power are the same, the increase of the temperature in time should be identical. The maximum temperature $T_{\max }$ at $t=$ $3 \cdot 10^{-4} \mathrm{~S}$ is slightly lower for the moving beam compared to the fixed beam with laser power $P=11.722 \mathrm{~kW} / \mathrm{m}$. During the diffusion phase, the difference in minimum and maximum temperature, $T_{\min }$ and $T_{\max }$ is smaller for the moving beam than for the fixed beam with laser power $P=11.722 \mathrm{~kW} / \mathrm{m}$, but the equilibrium value of the temperature $T_{\text {eq }}$ is similar. The behavior during the complete flow phase is the same for the moving beam and the fixed beam with laser power $P=11.722 \mathrm{~kW} / \mathrm{m}$, and therefore overlapping lines are shown in Figures 19(c-d).

In Figure 20, the temperature distribution (a-d) at different times within the startup phase, (e) at the end of the diffusion phase, and (f) at the end of the complete flow phase for the moving laser beam is shown. During the startup phase, the spot with the temperature slowly moves to the right. At the end of the startup phase, the maximum temperature $T_{\max }$ is found at the boundary at approximately $x=3 R / 2$ of the top half. At the end of the diffusion phase, the highest temperatures are found below the boundary close to $x=R$ of the top half. The slightly asymmetric temperature distribution at the end of the startup and diffusion phases is due to the asymmetric conductive heat transfer inside the domain and heat losses due to the movement of the beam from left to right. The maximum temperature $T_{\max }$ is located at the center of the system $(x, y)=(0,0)$ at the end of the complete flow phase.

From the results in this section, we find that the differences between a moving beam and a static beam at position $x_{\mathrm{b}}=0$ are small in this model system. 


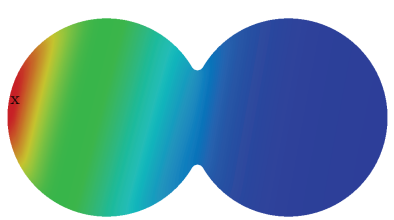

(a)
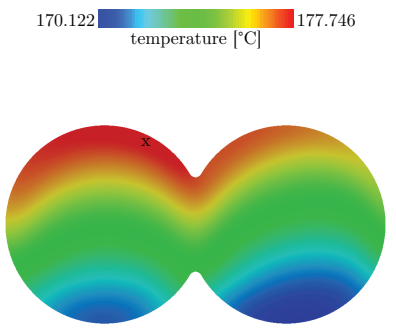

(c)

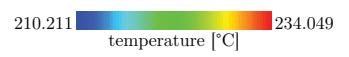

(e)
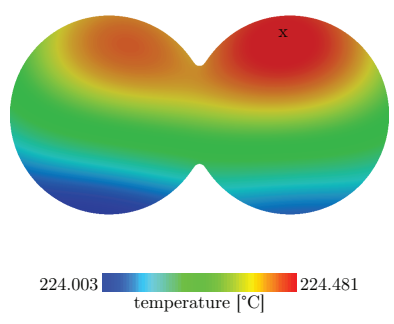

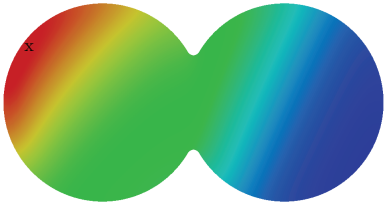

(b)
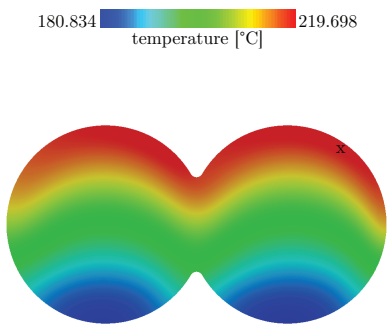

(d)
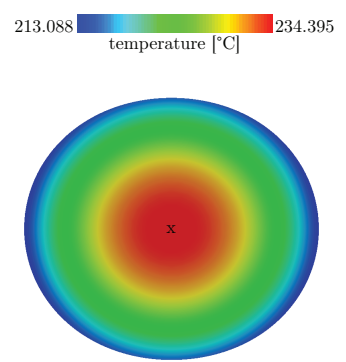

186.920 temperature $\left[{ }^{\circ} \mathrm{C}\right]$

Figure 20: Temperature distribution of the moving laser beam at (a) $t=0.5 \cdot 10^{-4} \mathrm{~s}$, (b) $t=1.5 \cdot 10^{-4} \mathrm{~s}$, (c) $t=2.5 \cdot 10^{-4} \mathrm{~s}$, (d) the end of the startup phase, (e) the end of the diffusion phase, and (f) the end of the complete flow phase $(t=2.28 \mathrm{~s})$. Note that the ranges of the color bars are different for each subfigure, to emphasize the temperature distribution for each phase. The ' $x$ ' marks the location of the maximum temperature $T_{\max }$.

\section{Conclusions}

We studied the temperature-dependent sintering process of polymer powder for additive manufacturing (SLS). For this, we developed a numerical model in which the flow and temperature problems are solved using the finite element method for fluids with the Newtonian constitutive behavior for purely viscous fluids on a $2 \mathrm{D}$ geometry of two circular particles, initially connected by a certain contact line.

By analyzing the dimensionless groups in the problem, we found that the heat produced by viscous dissipation is negligible, and that the laser source term is dominant in the energy balance. The temperature increase due to the addition of energy by the laser beam is calculated analytically using a simplified version of the geometry to compute the power losses. This result is compared to the temperature increase found using our computational model. The analytical value and the numerical result are in good agreement.

Because of the large amount of variables in the set of equations for the sintering problem, we restricted ourselves to assess only the easily tunable process parameters, and kept most of the material parameters constant. The following conclusions from our 2D model system are most relevant for the sintering process: The temperature $T$ in the system increases for increasing laser power $P$, decreasing beam width $W$, increasing 
ambient temperature $T_{\infty}$, and a beam position $x_{\mathrm{b}}$ closer to the center of the system, leading to a lower viscosity $\mu(T)$, a faster increase in contact line $L$, and a longer time to reach the melting temperature $T_{\mathrm{m}}$. These properties are advantageous for the formation of a homogeneous melt. On the other hand, with increasing temperature, the cooling down takes longer, which is disadvantageous for the speed of the sintering process. The cooling rate depends, besides on the convection coefficient $h$, largely on the ambient temperature $T_{\infty}$, which should therefore be as low as possible for a fast sintering process. However, it cannot be too low, because of crystallization and associated shrinkage. To reach the necessary increase in temperature, either the laser power $P$ should be increased, or the beam width $W$ should be decreased. Decreasing the beam width $W$ might help increase the resolution of the products. From the flow perspective, it would be beneficial if the viscosity $\mu(T)$ is as low as possible just above the melting temperature $T_{\mathrm{m}}$. This leads to a fast fusion, while the increase in temperature of the system is limited.

Finally, we let the laser beam move over the particles. This results in a slightly asymmetric temperature distribution. The behavior of both the maximum temperature $T_{\max }$ and the contact line $L$ in time is comparable with a static beam delivering the same amount of energy in this model system.

Future work within the group is focused on extending the presented computational model to 3D, and developing an experimental setup to analyze the sintering of two individual particles, and to compare the experimental results with the model.

\section{Acknowledgements}

This work is part of the Additive Manufacturing program of the Brightlands Materials Center.

\section{References}

\section{References}

[1] S. Kou and Y.H. Wang. Three-dimensional convection in laser melted pools. Metallurgical Transactions A, 17(12):2265-2270, 1986.

[2] N.K. Tolochko, M.K. Arshinov, A.V. Gusarov, V.I. Titov, T. Laoui, and L. Froyen. Mechanisms of selective laser sintering and heat transfer in $\mathrm{Ti}$ powder. Rapid Prototyping Journal, 9(5):314-326, 2003.

[3] W.D. Kingery. Introduction to ceramics. John Wiley \& Sons, New York, NY, 1960.

[4] R.B. Patil and V. Yadava. Finite element analysis of temperature distribution in single metallic powder layer during metal laser sintering. International Journal of Machine Tools and Manufacture, 47(7):1069-1080, 2007.

[5] T. Chen and Y. Zhang. Thermal modeling of laser sintering of two-component metal powder on top of sintered layers via multi-line scanning. Applied Physics A, 86(2):213-220, 2007.

[6] T. Chen and Y. Zhang. Three-dimensional modeling of laser sintering of a twocomponent metal powder layer on top of sintered layers. Journal of Manufacturing Science and Engineering, 129(3):575-582, 2007.

[7] I.A. Roberts, C.J. Wang, R. Esterlein, M. Stanford, and D.J. Mynors. A threedimensional finite element analysis of the temperature field during laser melting of metal powders in additive layer manufacturing. International Journal of Machine Tools and Manufacture, 49(12):916-923, 2009. 
[8] L.P. Kholpanov, S.E. Zakiev, and I.V. Shishkovskii. Modeling of thermal processes in laser sintering of reactive powder compositions. Journal of Engineering Physics and Thermophysics, 78(6):1088-1095, 2005.

[9] S.E. Zakiev, L.P. Kholpanov, I.V. Shishkovsky, I.P. Parkin, M.V. Kuznetsov, and Y.G. Morozov. Modelling of the thermal processes that occur during laser sintering of reacting powder compositions. Applied Physics A, 84(1-2):123-129, 2006.

[10] S. Kolossov, E. Boillat, R. Glardon, P. Fischer, and M. Locher. 3D FE simulation for temperature evolution in the selective laser sintering process. International Journal of Machine Tools and Manufacture, 44(2):117-123, 2004.

[11] J. Frenkel. Viscous flow of crystalline bodies under the action of surface tension. Journal of Physics IX, 5:385-391, 1945.

[12] G.W. Scherer. Sintering of low-density glasses: I, theory. Journal of the American Ceramic Society, 60(5-6):236-239, 1977.

[13] M. Kandis and T.L. Bergman. A simulation-based correlation of the density and thermal conductivity of objects produced by laser sintering of polymer powders. Journal of Manufacturing Science and Engineering, 122(3):439-444, 2000.

[14] M.M. Sun, J.C. Nelson, J.J. Beaman, and J.W. Barlow. A model for partial viscous sintering. In Proceedings of Solid Freeform Fabrication Symposium Austin, TX, 1991.

[15] M.M. Sun and J.J. Beaman. A three dimensional model for selective laser sintering. In Proceedings of Solid Freeform Fabrication Symposium Austin, TX, 1991.

[16] A.L. Papadatos, S. Ahzi, and C.R. Deckard F.W. Paul. On dimensional stabilities: modelling of the bonus-z during the SLS process. In Proceedings of Solid Freeform Fabrication Symposium Austin, TX, 1997.

[17] J.K. Mackenzie and R. Shuttleworth. A phenomenological theory of sintering. Proceedings of the Physical Society B, 62(12):833-852, 1949.

[18] J.C. Nelson, S. Xue, J.W. Barlow, J.J. Beaman, H.L. Marcus, and D.L. Bourell. Model of the selective laser sintering of bisphenol-A polycarbonate. Industrial and Engineering Chemistry Research, 32(10):2305-2317, 1993.

[19] G. Bugeda, M. Cervera, and G. Lombera. Numerical prediction of temperature and density distributions in selective laser sintering processes. Rapid Prototyping Journal, $5(1): 21-26,1999$.

[20] A.K. Singh and R. Srinivasa Prakash. Response surface-based simulation modeling for selective laser sintering process. Rapid Prototyping Journal, 16(6):441-449, 2010.

[21] T.H.C. Childs, M. Berzins, G.R. Ryder, and A. Tontowi. Selective laser sintering of an amorphous polymer - simulations and experiments. Proceedings of the Institution of Mechanical Engineers, Part B: Journal of Engineering Manufacture, 213(4):333-349, 1999 .

[22] L. Dong, A. Makradi, S. Ahzi, and Y. Remond. Three-dimensional transient finite element analysis of the selective laser sintering process. Journal of Materials Processing Technology, 209(2):700-706, 2009.

[23] A.E. Tontowi and T.H.C. Childs. Density prediction of crystalline polymer sintered parts at various powder bed temperatures. Rapid Prototyping Journal, 7(3):180-184, 2001. 
[24] K. Zeng, D. Pal, and B. Stucker. A review of thermal analysis methods in laser sintering and selective laser melting. In Proceedings of Solid Freeform Fabrication Symposium Austin, TX, 2012.

[25] R. Hooper, C.W. Macosko, and J.J. Derby. Assessing a flow-based finite element model for the sintering of viscoelastic particles. Chemical Engineering Science, $55(23): 5733-5746,2000$.

[26] C. Balemans, M.A. Hulsen, and P.D. Anderson. Sintering of two viscoelastic particles: a computational approach. Applied Sciences, 7(5):516, 2017.

[27] L. Verbelen, S. Dadbakhsh, M. Van den Eynde, J.P. Kruth, B. Goderis, and P. Van Puyvelde. Characterization of polyamide powders for determination of laser sintering processability. European Polymer Journal, 75:163-174, 2016.

[28] J. Brandrup, E.H. Immergut, and E.A. Grulke. Polymer Handbook, 4th edition. John Wiley \& Sons, New York, NY, 1999.

[29] M.M. Villone, M.A. Hulsen, P.D. Anderson, and P.L. Maffettone. Simulations of deformable systems in fluids under shear flow using an arbitrary Lagrangian Eulerian technique. Computers $\mathscr{G}$ Fluids, 90:88-100, 2014.

[30] C.E. Weatherburn. Differential Geometry of Three Dimensions. Cambridge University Press, Cambridge, 1955.

[31] C. Geuzaine and J.F. Remacle. Gmsh: a three-dimensional finite element mesh generator with built-in pre- and post-processing facilities. International Journal for Numerical Methods in Engineering, 79(11):1309-1331, 2009.

[32] N.O. Jaensson, M.A. Hulsen, and P.D. Anderson. Stokes-Cahn-Hilliard formulations and simulations of two-phase flows with suspended rigid particles. Computers $\&$ Fluids, 111:1-17, 2015.

[33] R.W. Hopper. Plane Stokes flow driven by capillarity on a free surface. Journal of Fluid Mechanics, 213:349-375, 1990.

[34] M. Yuan, D. Bourell, and T. Diller. Thermal conductivity measurements of polyamide 12. In Proceedings of the Solid Freeform Fabrication Symposium Austin, TX, pages 427-437, 2011.

[35] T. Laumer, T. Stichel, K. Nagulin, and M. Schmidt. Optical analysis of polymer powder materials for Selective Laser Sintering. Polymer Testing, 56:207-213, 2016.

[36] T.T. Diller, R. Sreenivasan, J. Beaman, D. Bourell, and J. LaRocco. Thermal model of the build environment for polyamide powder selective laser sintering. In Proceedings of Solid Freeform Fabrication Symposium Austin, TX, pages 539-548, 2010. 